\title{
HEAT KERNELS ON HOMOGENEOUS SPACES
}

\author{
C. M. P. A. SMULDERS
}

(Received 15 January 2001; revised 13 August 2003)

Communicated by A. H. Dooley

\begin{abstract}
Let $a_{\mathfrak{i}}, \ldots, a_{d}$ be a basis of the Lie algebra $g$ of a connected Lie group $G$ and let $M$ be a Lie subgroup of. $G$. If $d x$ is a non-zero positive quasi-invariant regular Borel measure on the homogeneous space $X=G / M$ and $S: X \times G \rightarrow \mathbb{C}$ is a continuous cocycle, then under a rather weak condition on $d x$ and $S$ there exists in a natural way a (weakly*) continuous representation $U$ of $G$ in $L_{p}(X ; d x)$ for all $p \in[1, \infty]$.

Let $A_{i}$ be the infinitesimal generator with respect to $U$ and the direction $a_{i}$ for all $i \in\{1, \ldots, d\}$. We consider $n$-th order strongly elliptic operators $H=\sum c_{\alpha} A^{\alpha}$ with complex coefficients $c_{\alpha}$. We show that the semigroup $S$ generated by the closure of $H$ has a reduced heat kernel $\kappa$ and we derive upper bounds for $K$ and all its derivatives.
\end{abstract}

2000 Mathematics subject classification: primary 43A85, 22D30, 22E25, 22E45, 35K05.

Keywords and phrases: reduced heat kernel, homogeneous space, quasi-invariant measure, Gaussian estimate, strongly elliptic operator, cocycle representation.

\section{Introduction}

We analyze Gaussian bounds for the heat kernel of the semigroup generated by a strongly elliptic operator on a homogeneous space. Gaussian estimates for kernels associated to various kinds of semigroup generators have been deduced, amongst others, for second-order elliptic operators on domains in $\mathbb{R}^{n}$, by Davies [3]; for the Laplace-Beltrami operator on complete Riemannian manifolds by Li and Yau in [11]; for the Laplace-Beltrami operator on a complete Riemannian manifold with nonnegative Ricci curvature by Davies [3] and Grigor'yan [8]; on a non-compact manifold with Ricci curvature bounded from below by Grigor'yan [8]; for subelliptic operators which are sums of squares of Hörmander vector fields on compact manifolds endowed

(C) 2005 Australian Mathematical Society $1446-7887 / 05 \$ A 2.00+0.00$ 
with a Radon measure by Jerison and Sanchez-Callé [9]; for weighted subcoercive operators on a Lie group [5] and for strongly elliptic operators on a homogeneous space $G / M$, with $G$ unimodular and $M$ compact, endowed with the $G$-invariant measure on $G / M$ [6]; for elliptic operators on Lie groups using Harnack inequalities by Varopoulos in [18]; for sublaplacians on nilmanifolds and homogeneous spaces $X=G / M$ with $G$ and $M$ unimodular by Maheux [14]; for elliptic operators on amenable Lie groups and certain homogeneous spaces by Lohoué and Mustapha in [12]; for elliptic operators on Euclidean spaces by Aronson [2]. Our aim is to deduce Gaussian estimates for the heat kernel associated to a strongly elliptic operator on a general homogeneous space $G / M$ endowed with a non-zero positive quasi-invariant regular Borel measure. The strongly elliptic operators are affiliated to the natural representation corresponding to this quasi-invariant measure, a continuous cocycle $S: X \times G \rightarrow \mathbb{C}$ and translations. Note that the Lie groups $G$ and $M$ need not be unimodular and $M$ is allowed to be disconnected but still $\sigma$-compact. Further note that the class of operators and the class of representations considered in this paper are quite large. The Radon measures considered in [9] are replaced by quasi-invariant measures in this paper.

If $\kappa$ is the heat kernel of the semigroup generated by the Laplace-Beltrami operator on a complete non-compact Riemannian manifold $X$ with Ricci curvature bounded from below then Grigor'yan proved that there exist $a, b, \omega>0$ such that

$$
\kappa_{t}(x ; y) \leq a t^{-N / 2} e^{\omega t}\left(\operatorname{Vol}_{X}(B(x ; 1)) \operatorname{Vol}_{X}(B(y ; 1))\right)^{-1 / 2} e^{-b d(x ; y)^{2} t^{-1}}
$$

uniformly for all $x, y \in X$ and $t>0$, where $d$ denotes the Riemannian metric on $X$ and $N$ is the dimension of $X$ (see, for example, [8, page 445] and [11]). On the other hand, in [6] we derived Gaussian bounds for the heat kernel $\kappa$ of the semigroup generated by an $n$-th order strongly elliptic operator affiliated to the left regular representation of a unimodular Lie group $G$ in a homogeneous space $X=G / M$, with $M$ compact, endowed with the $G$-invariant measure on $X$. More precisely, there exist $a, b, \omega>0$ such that

$$
\left|\kappa_{t}(x ; y)\right| \leq a t^{-N / n} e^{\omega t} e^{-b\left(d(x ; y)^{n} t^{-1}\right)^{1 /(n-1)}}
$$

uniformly for all $x, y \in X$ and $t>0$, where $d$ is the path distance on $X$ associated to the vector fields induced by the left regular representation of $G$ on $X$ and $N$ is the dimension of $X$. In this paper we drop the assumptions on $G$ and $M$ and (therefore also) the $G$-invariance of the measure on $X$ (see also [12]). For the sublaplacian on homogeneous spaces $X=G / M$ with $G$ and $M$ unimodular, similar Gaussian bounds were derived in [14]. Let $d x$ be a quasi-invariant measure on $X$. Under a rather weak condition on the measure $d x$ there is a continuous representation of $G$ in $L_{2}(X ; d x)$, which can be extended to all the $L_{p}$-spaces. In the main theorem of this paper we 
prove that the semigroup generated by an $n$-th order strongly elliptic operator has a heat kernel $\kappa$ and there exist $a, b, \omega>0$ such that

$$
\left|\kappa_{t}(x ; y)\right| \leq a t^{-N / n} e^{\omega t}\left(\operatorname{Vol}_{X}(B(x ; 1)) \operatorname{Vol}_{X}(B(y ; 1))\right)^{-1 / 2} e^{-b\left(d(x ; y)^{n} t^{-1}\right)^{1 /(n-1)}}
$$

uniformly for all $x, y \in X$ and $t>0$, where $d$ denotes the path distance on $X$ associated to the vector fields induced by the left regular representation of $G$ on $X, N$ is the dimension of $X, B(x ; 1)$ is the $d$-ball with center $x$ and radius 1 and the volumes are with respect to the quasi-invariant measure $d x$. Compare these upper bounds with the kernel estimates deduced in $[12,14]$. In general, the volume factor

$$
\left(\operatorname{Vol}_{X}(B(x ; 1)) \operatorname{Vol}_{X}(B(y ; 1))\right)^{-1 / 2}
$$

in (3) is necessary and bounds of the form (2) are not valid in general. There already exist examples of quasi-invariant measures $d x$ on $\mathbb{R}$ for which (2) does not hold whereas (3) is still valid. Alternatively, the bounds (3) are in general sharper than the bounds in the situation of (2). Note that one has an exponent $-1 / 2$ in the volume factor, even for $n$-th order operators, as in the upper bounds (1) of Grigor'yan and $\mathrm{Li}$ and Yau.

The infinitesimal generators associated to the continuous representation are sums of a vector field and a potential. The potential is caused by the quasi-invariance of the measure $d x$ on $X$ and the cocycle $S$.

The proof of (3) for the Laplacian is via a Nash inequality and for higher order operators with the aid of a reduction formula. The Nash inequality, which we deduce in Section 4 via a Young inequality, involves a quotient between $r^{N}$ and the volume of balls of radius $r$, where $N$ denotes the dimension of $X$ again. In Section 3 we deduce a scaling property which determines this quotient. The reduction procedure to obtain the Gaussian bounds (3) for all (higher) order operators is established in Section 5. Moreover, we deduce Gaussian bounds and reduction formulas for all derivatives of the heat kernel $\kappa$.

\section{Preliminary notation and main results}

In this section we introduce some preliminary notation and conditions which ensure the existence of nice representations and reduced heat kernels. We also state the main results of the paper. The results of this section are from [16, Chapter 2].

Throughout this paper let $G$ be a $d$-dimensional connected (possibly non-unimodular) Lie group with (left) Haar measure $d g$ and modular function $\Delta_{G}$ and $M$ a

$d_{M}$-dimensional Lie subgroup with (left) Haar measure $d m$ and modular function $\Delta_{M}$. Note that $G$ and $M$ are both closed (possibly non-unimodular) $\sigma$-compact topological 
groups, and that $M$ is not necessarily connected. Consider the homogeneous space $X=G / M$. For all $g \in G$ we denote by $\dot{g}$ the left coset $g M$. Let $d x=d \dot{g}$ be a $\sigma$-finite quasi-invariant non-zero regular Borel measure on $X$, that is, $d x$ has the same null sets as all the left translates of $d x$. If $X$ is compact then $d x$ is a finite measure.

By the Radon-Nikodým theorem, there exists a function $R: X \times G \rightarrow\langle 0, \infty\rangle$ such that for each $g \in G$ the function $x \mapsto R(x, g)$ is Borel measurable, for all $\varphi \in C_{c}(X)$ the function $x \mapsto \varphi(x) R(x, g)$ belongs to $L_{1}(X ; d x)$, and,

$$
\int_{X} \varphi\left(g^{-1} x\right) d x=\int_{X} \varphi(x) R(x, g) d x .
$$

In the sequel we only consider quasi-invariant measures $d x$ for which there exists a continuous function $R: X \times G \rightarrow\langle 0, \infty\rangle$ such that

$$
\int_{X} \varphi\left(g^{-1} x\right) d x=\int_{X} \varphi(x) R(x, g) d x
$$

for all $g \in G$ and $\varphi \in C_{c}(X)$. For each $g \in G$, the function $x \mapsto R(x, g)$ is bounded from above and bounded away from 0 on compacta in $X$, and hence the right-hand side of (4) makes sense for all $\varphi \in C_{c}(X)$. Then it follows that

$$
R(x, g h)=R(h x, g) R(x, h) \text { and } R(x, g)^{-1}=R\left(g x, g^{-1}\right)
$$

for all $x \in X$ and $g, h \in G$, and therefore $R(x, e)=1$ for all $x \in X$. The relations (5) mean that $R$ is a cocycle.

Let $a_{1}, \ldots, a_{d}$ be a fixed basis of the Lie algebra $\mathfrak{g}$ of $G$ such that $a_{1}, \ldots, a_{d_{M}}$ is a basis for the Lie algebra $m$ of $M$. The modulus $|\cdot|$ on the Lie group $G$ is defined by

$$
|g|=\inf \int_{0}^{l}\left(\sum_{i=1}^{d} \gamma_{i}^{2}(t)\right)^{1 / 2} d t
$$

where the infimum is taken over the set of all absolutely continuous paths

$$
\gamma:[0,1] \rightarrow G \text { such that } \gamma(0)=e, \gamma(1)=g, \text { and } \dot{\gamma}(t)=\left.\sum_{i=1}^{d} \gamma_{i}(t) \tilde{A}_{i}\right|_{\gamma(t)}
$$

for a.e. $i \in[0,1]$, where $\widetilde{A_{1}}, \ldots, \widetilde{A_{d}}$ denote the vector fields induced by the infinitesimal generators in the directions $a_{1}, \ldots, a_{d}$ with respect to the left regular representation of $G$ in $L_{\infty}(G ; d g)$. The modulus on $M$, denoted by $|\cdot|_{M}$, is defined analogously. Let $B_{\varepsilon}=\{g \in G:|g|<\varepsilon\}$ and $B_{\varepsilon, M}=\left\{m \in M:|m|_{M}<\varepsilon\right\}$ be the corresponding balls for all $\varepsilon>0$.

In order to construct continuous representations leaving all $L_{p}$-spaces invariant, and to define reduced heat kernels, in the sequel we demand $R$ to satisfy the following condition. 
There exists a $C_{R} \geq 1$ such that

$$
C_{R}^{-1} \leq R(x, g) \leq C_{R}
$$

for all $x \in X$ and $g \in B_{1}$.

Throughout this paper, let $C_{R}$ be the constant as in (6). Clearly, (6) is valid if $X$ is compact. It is easy to show that there exist $C, \eta>0$ such that

$$
C^{-1} e^{-\eta|g|} \leq R(x, g) \leq C e^{\eta|g|}
$$

for all $x \in X$ and $g \in G$.

The following lemma will be used frequently in this paper.

LEMMA 2.1. If $g \in G$ and $\varphi: X \rightarrow \mathbb{C}$ is a measurable function then the following conditions are equivalent:

(I) $\varphi \in L_{1}(X ; d x)$.

(II) $x \mapsto \varphi\left(g^{-1} x\right)$ belongs to $L_{1}(X ; d x)$.

(III) $x \mapsto \varphi(x) R(x, g)$ belongs to $L_{1}(X ; d x)$.

Moreover, if one of the three conditions is satisfied then (4) is valid.

PROOF. The lemma follows from (4), (7) and approximation.

The following two lemmas play a key role in this paper. For proofs we refer to [10, Lemma 3.3, Lemma 3.10, Theorem 3.11] and [10, remark on page 349].

LEMMA 2.2. Given the measure $d x$ and the corresponding continuous function $R$, one can construct a unique continuous function $\rho: G \rightarrow\langle 0, \infty\rangle$ with the following four properties:

(I) If $g \in G$ and $m \in M$, then

$$
\frac{\rho(g m)}{\rho(g)}=\frac{\Delta_{M}(m)}{\Delta_{G}(m)} .
$$

(II) If $\varphi: G \rightarrow[0, \infty)$ is measurable, then the function $\Phi: X \mapsto[0, \infty]$ given by $\Phi(\dot{g})=\int_{M} \varphi(g m) d m(g \in G)$ is well defined, and $\varphi \in L_{\mathrm{i}}(G ; \rho d g)$ if and only if $\Phi \in L_{1}(X ; d x)$. Moreover, if $\varphi \in L_{1}(G ; \rho d g)$, then

$$
\int_{G} \varphi(g) \rho(g) d g=\int_{X} \Phi(x) d x .
$$

(III) The function $\rho$ satisfies the identity

$$
R(\dot{k}, g)=\frac{\rho(g k)}{\rho(k)}
$$

for all $g, k \in G$. 
LEMMA 2.3. Let $M$ be a Lie subgroup of a connected Lie group $G$ with modular functions $\Delta_{M}$ and $\Delta_{G}$, respectively. Then the following two statements hold.

(I) If $\rho: G \rightarrow\langle 0, \infty\rangle$ is a continuous function, which satisfies (8), and $R$ is defined as in (10), then there exists a (up to constant) unique quasi-invariant regular Borel measure $d x$ on $X$ satisfying (4).

(II) There exists a (in general not unique) $C^{\infty}$-function $\rho: G \rightarrow\langle 0, \infty\rangle$ which satisfies (8).

Let $S: X \times G \rightarrow \mathbb{C}$ be a continuous cocycle, that is, $S(x, g h)=S(h x, g) S(x, h)$ and $S(x, e)=1$ for all $g, h \in G$ and $x \in X$. Throughout this paper, we assume that there is a $C_{S} \geq 1$ such that

$$
C_{S}^{-1} \leq|S(x, g)| \leq C_{S}
$$

for all $x \in X$ and $g \in B_{1}$. Note the similarity between $S$ and $R$. There exist $C^{\prime}, \eta^{\prime}>0$ such that

$$
\left(C^{\prime}\right)^{-1} e^{-\eta^{\prime}|g|} \leq|S(x, g)| \leq C^{\prime} e^{\eta^{\prime}|g|}
$$

for all $x \in X$ and $g \in G$. By (7), (12) and Lemma 2.1 we can introduce for all $p \in[1, \infty]$ the representation $U$ of $G$ in $L_{p}(X ; d x)$ by

$$
\begin{aligned}
(U(g) \varphi)(x) & =S\left(g^{-1} x, g\right) R\left(x, g^{-1}\right)^{1 / 2} \varphi\left(g^{-1} x\right) \\
& =S\left(x, g^{-1}\right)^{-1} R\left(x, g^{-1}\right)^{1 / 2} \varphi\left(g^{-1} x\right)
\end{aligned}
$$

for all $\varphi \in L_{p}(X ; d x)$.

Since the map $g \mapsto U(g)$ is locally bounded on each $L_{p}(X ; d x)$ with $p \in[1, \infty]$, and $C_{c}(X)$ is dense in each $L_{p}(X ; d x)$, one deduces the following proposition for $p \in[1, \infty]$. The case $p=\infty$ is obtained by duality.

PROPOSITION 2.4. The representation $U$ is strongly continuous in $L_{p}(X ; d x)$ for all $p \in[1, \infty)$ and weakly* continuous in $L_{\infty}(X ; d x)$. Moreover, $U$ is a unitary representation in $L_{2}(X ; d x)$ if $|S|=1$.

For $i \in\{1, \ldots, d\}$, let $A_{i}=d U\left(a_{i}\right)$ denote the infinitesimal generator in the direction $a_{i}$ affiliated to the representation $U$, that is, the infinitesimal generator of the one parameter group $t \mapsto U\left(\exp \left(-t a_{i}\right)\right)$. We also need multi-indices notation. For $N \in \mathbb{N}$, let $J(N)=\bigoplus_{k=0}^{\infty}\{1, \ldots, N\}^{k}$ denote the set of all multi-indices over the index set $\{1, \ldots, N\}$. If $\alpha=\left(i_{1}, \ldots, i_{k}\right) \in J(d)$ then we set $A^{\alpha}=A_{i_{1}} \circ \cdots \circ A_{i_{k}}$, and, we denote by $|\alpha|=k$ the length of the multi-index $\alpha$.

Let $n \in \mathbb{N}$ be even, and for all $\alpha \in J(d)$, with $|\alpha| \leq n$, let $c_{\alpha} \in \mathbb{C}$. We consider the operator $H=\sum_{\alpha \in J(d):|\alpha| \leq n} c_{\alpha} A^{\alpha}$, affiliated to the representation $U$ in (13), and with 
domain $D(H)=\bigcap_{\alpha \in J(d):|\alpha| \leq n} D\left(A^{\alpha}\right)$. The operator $H$ is called an $n$-th order strongly elliptic operator if there is a $\mu>0$ such that $\operatorname{Re}(-1)^{n / 2} \sum_{\alpha \in J(d):|\alpha|=n} c_{\alpha} \xi^{\alpha} \geq|\xi|^{n}$ for all $\xi \in \mathbb{R}^{d}$, where $\xi^{\alpha}=\xi_{i_{1}} \cdots \xi_{i_{k}}$ for all $\alpha=\left(i_{1}, \ldots, i_{k}\right) \in J(d)$. By [15, Theorem I.5.1], the closure of $H$ generates a continuous semigroup $S=S_{t}$. Moreover, for all $t>0$ there exists a smooth fast decaying Lie group kernel $K_{f} \in L_{1}(G ; d g)$ such that $S_{t} \varphi=\int_{G} K_{t}(g) U(g) \varphi d g$ for all $\varphi \in \bigcup_{p=1}^{\infty} L_{p}(X ; d x)$.

In order to show that the semigroup $S=S_{t}$ generated by $\bar{H}$ has a continuous heat kernel $k: X \times X \rightarrow \mathbb{C}$ we need some preparation. The following lemma will be applied several times in this paper.

LEMMA 2.5. Let $f: G \rightarrow \mathbb{C}$ be a measurable function and $a, b>0$ such that $|f(g)| \leq a e^{-b|g|^{n /(n-1)}}$ for all $g \in G$. Then the following three statements are valid:

(I) The integral

$$
\int_{M} f\left(g m^{-1} k^{-1}\right) S\left(\dot{k}, g m^{-1} k^{-1}\right)(\rho(g) \rho(k m))^{-1 / 2} \Delta_{G}\left(m^{-1} k^{-1}\right) d m
$$

exists for all $g, k \in G$.

(II) There exists a function $F: X \times X \rightarrow \mathbb{C}$ such that

$$
F(\dot{g}, \dot{k})=\int_{M} f\left(g m^{-1} k^{-1}\right) S\left(\dot{k}, g m^{-1} k^{-1}\right)(\rho(g) \rho(k m))^{-1 / 2} \Delta_{G}\left(m^{-1} k^{-1}\right) d m
$$

for all $g, k \in G$.

(III) If $Y$ is a compact subset of $X$ then the function $F$ is bounded on $Y \times Y$.

PROOF. There exist $C, \eta>0$ such that

$$
\Delta_{G}\left(m^{-1} k^{-1}\right) \leq C e^{\eta|k m|}
$$

for all $k \in G$ and $m \in M$. Moreover, since $\rho(k m)^{-1}=\rho(e)^{-1} R(\dot{e}, k m)^{-1}$, it follows from (7) that there exist $C, \eta>0$ such that

$$
\rho(k m)^{-1} \leq C e^{\eta|k m|}
$$

for all $k \in G$ and $m \in M$. Further, it follows from (12) that

$$
\left|S\left(\dot{k}, g m^{-1} k^{-1}\right)\right| \leq C^{\prime} e^{\eta^{\prime}|g|} e^{\eta^{\prime}|k m|}
$$

for all $g, k \in G$ and $m \in M$.

Now, let $g \in G$. Since $|k m| \leq\left|g m^{-1} k^{-1}\right|+|g|$ for all $m \in M$ and $k \in G$, and $(a+b)^{\lambda} \leq 2^{\lambda}\left(a^{\lambda}+b^{\lambda}\right)$ for all $\lambda>0$ and $a, b>0$, it follows that

$$
2^{-n /(n-1)}|k m|^{n /(n-1)} \leq\left|g m^{-1} k^{-1}\right|^{n /(n-1)}+|g|^{n /(n-1)}
$$


for all $m \in M$ and $k \in G$. Therefore, it follows from (15), (16) and (17) that there exist $a^{\prime}, b^{\prime}>0$ such that

$$
\begin{gathered}
\int_{M}\left|f\left(g m^{-1} k^{-1}\right) S\left(\dot{k}, g m^{-1} k^{-1}\right)\right|(\rho(g) \rho(k m))^{-1 / 2} \Delta_{G}\left(m^{-1} k^{-1}\right) d m \\
\leq a^{\prime} e^{b|g|^{n /(n-1)}} e^{\eta^{\prime}|g|} \rho(g)^{-1 / 2} \int_{M} e^{-b^{\prime}|k m|^{n /(n-1)}} d m
\end{gathered}
$$

for all $k \in G$. Statement (I) is proved, if we can show that $\Phi(\dot{k})<\infty$ for all $k \in G$, where

$$
\Phi(\dot{k})=\int_{M} e^{-b^{\prime}|k m|^{n /(n-1)}} d m
$$

for all $k \in G$. Let $k \in G$. It is clear that

$$
\begin{aligned}
|m|^{n /(n-1)} & \leq(|k m|+|k|)^{n /(n-1)} \leq 2^{n /(n-1)}|k m|^{n /(n-1)}+2^{n /(n-1)}|k|^{n /(n-1)} \\
& \leq 4|k m|^{n /(n-1)}+4|k|^{n /(n-1)}
\end{aligned}
$$

for all $m \in M$. Therefore,

$$
e^{-b^{\prime}|k m|^{n /(n-1)}} \leq e^{-4^{-1} b^{\prime}|m|^{n /(n-1)}} e^{b^{\prime}|k|^{n /(n-1)}}
$$

for all $m \in M$. It follows that

$$
\Phi(\dot{k}) \leq e^{b^{\prime}|k|^{n /(n-1)}} \int_{M} e^{-4^{-1} b^{\prime}|m|^{n /(n-1)}} d m .
$$

We next show that there is an $\eta>0$ such that $\operatorname{Vol}_{M}\left(M \cap B_{r}\right) \leq e^{\eta r}$ for all $r \geq 1$. Let $r \geq 1$. Then there exist $N \in \mathbb{N}$ and $g_{1}, \ldots, g_{N} \in G$ such that $g_{1} B_{1 / 4}, \ldots, g_{N} B_{1 / 4}$ is a maximal set of disjoint balls with radii $4^{-1}$ in $B_{2 r}$. Suppose that there is a $g \in B_{r}$ such that no $g_{1}, \ldots, g_{N}$ is contained in $g B_{1 / 2}$. If $i \in\{1, \ldots, N\}$ and $h \in g_{i} B_{1 / 4}$, then

$$
\left|g^{-1} h\right| \geq\left|g^{-1} g_{i}\right|-\left|g_{i}^{-1} h\right|>2^{-1}-4^{-1}=4^{-1},
$$

and $g B_{1 / 4} \subseteq B_{2 r}$ is disjoint from all $g_{1} B_{1 / 4}, \ldots, g_{N} B_{1 / 4}$. Therefore, $g_{1} B_{1 / 2}, \ldots$, $g_{N} B_{1 / 2}$ cover $B_{r}$. If $\alpha=\operatorname{Vol}_{G}\left(B_{1 / 4}\right)$, then it follows from the disjointness of the balls $g_{1} B_{1 / 4}, \ldots, g_{N} B_{1 / 4}$, and the left invariance of $d g$, that

$$
N \alpha \leq \operatorname{Vol}_{G}\left(B_{2 r}\right)
$$

But, there exists a $\theta>0$ such that $\operatorname{Vol}_{G}\left(B_{s}\right) \leq e^{\theta s}$ for all $s \geq 1$. Hence, $N \leq \alpha^{-1} e^{2 \theta r}$. Furthermore,

$$
M \cap B_{r} \subseteq M \cap \bigcup_{i=1}^{N} g_{i} B_{1 / 2}=\bigcup_{i=1}^{N}\left(M \cap g_{i} B_{1 / 2}\right)
$$


If $i \in\{1, \ldots, N\}, m_{0} \in M \cap g_{i} B_{1 / 2}$ and $m \in M \cap g_{i} B_{1 / 2}$, then $m_{0}^{-1} m \in M \cap B_{1 / 2}^{-1} B_{1 / 2} \subseteq$ $M \cap B_{1}$, and hence $M \cap g_{i} B_{1 / 2} \subseteq m_{0}\left(M \cap B_{1}\right)$. Then it follows from the left invariance of the Haar measure $d m$, that there is an $\eta>0$, independent of $r$, such that

$$
\operatorname{Vol}_{M}\left(M \cap B_{r}\right) \leq N \operatorname{Vol}_{M}\left(M \cap B_{1}\right) \leq \operatorname{Vol}_{M}\left(M \cap B_{1}\right) \alpha^{-1} e^{2 \theta r} \leq e^{\eta r} .
$$

Hence, it follows from (20) that

$$
\begin{aligned}
\Phi(\dot{k}) & \leq e^{b^{\prime}|k|^{n /(n-1)}} \sum_{r=1}^{\infty} \int_{\{m \in M: r-1 \leq|m|<r\}} e^{-4^{-!} b^{\prime}(r-1)^{n /(n-1)}} d m \\
& \leq e^{b^{\prime}|k|^{n /(n-1)}} \sum_{r=1}^{\infty} \int_{M \cap B_{r}} e^{-4^{-1} b^{\prime}(r-1)^{n /(n-1)}} d m \\
& \leq e^{b^{\prime}|k|^{n /(n-1)}} \sum_{r=1}^{\infty} e^{\eta r} e^{-4^{-1} b^{\prime}(r-1)^{n /(n-1)}}<\infty,
\end{aligned}
$$

and hence $\Phi(\dot{k})<\infty$ for all $k \in G$, and Statement (I) follows immediately.

Next, one can define the function $\widetilde{F}: G \times G \rightarrow \mathbb{C}$ by

$$
\widetilde{F}(g, k)=\int_{M} f\left(g m^{-1} k^{-1}\right) S\left(\dot{k}, g m^{-1} k^{-1}\right)(\rho(g) \rho(k m))^{-1 / 2} \Delta_{G}\left(m^{-1} k^{-1}\right) d m
$$

for all $g, k \in G$. Since $\widetilde{F}\left(g m_{1}, k m_{2}\right)=\widetilde{F}(g, k)$ for all $g, k \in G$ and $m_{1}, m_{2} \in M$, the function $F: X \times X \rightarrow \mathbb{C}$, given by $F(\dot{g}, \dot{k})=\widetilde{F}(g, k)$ for all $g, k \in G$, is well defined, and Statement (II) follows directly.

If $Y$ is a compact subset of $X$ then, by [10, Lemma 2.4], there exists a compact subset $K$ of $G$ such that $K$ is mapped onto $Y$ by the natural projection map from $G$ onto $X=G / M$. Since $\widetilde{F}$ is bounded on $K \times K$, it follows that $F$ is bounded on $Y \times Y$, and Statement (III) follows. This completes the proof of the lemma.

By [15, Theorem III.4.8], there exist $a, b>0$ and $\omega \geq 0$ such that

$$
\left|K_{t}(g)\right| \leq a t^{-d / n} e^{\omega t} e^{-b\left(|g|^{n} t^{-1}\right)^{1 /(n-1)}}
$$

for all $g \in G$ and $t>0$. So, by Lemma 2.5 (I)-(II), one can define, for all $t>0$, the function $\kappa_{t}: X \times X \rightarrow \mathbb{C}$ by

$$
\kappa_{t}(\dot{g} ; \dot{k})=\int_{M} K_{t}\left(g m^{-1} k^{-1}\right) S\left(\dot{k}, g m^{-1} k^{-1}\right)(\rho(g) \rho(k m))^{-1 / 2} \Delta_{G}\left(m^{-1} k^{-1}\right) d m
$$

for all $g, k \in G$.

We call the function $\kappa_{t}$ the reduced heat kernel of the semigroup $S=S_{t}$ because of the following identity. 
PROPOSITION 2.6. If $p \in[1, \infty], \varphi \in L_{p}(X ; d x)$ and $t>0$, then

$$
\left(S_{t} \varphi\right)(x)=\int_{X} \kappa_{t}(x ; y) \varphi(y) d y
$$

for a.e. $x \in X$.

ProOF. Let $\psi, \varphi \in C_{c}(X)$ and $t>0$. Then

$$
\left(\psi, S_{t} \varphi\right)=\int_{G} K_{t}(g)(\psi, U(g) \varphi) d g=\int_{G} \int_{X} K_{t}(g) \overline{\psi(x)}(U(g) \varphi)(x) d x d g .
$$

Since $U$ is a continuous representation in $L_{2}(X ; d x)$, it follows from the CauchySchwartz inequality that there are $C \geq 1$ and $\eta>0$ such that

$$
\begin{aligned}
\int_{X}\left|K_{t}(g) \overline{\psi(x)}(U(g) \varphi)(x)\right| d x & \leq\left|K_{t}(g)\right|\|\psi\|_{2}\|U(g) \varphi\|_{2} \\
& \leq C e^{\eta|g|}\left|K_{t}(g)\right|\|\psi\|_{2}\|\varphi\|_{2}
\end{aligned}
$$

for all $g \in G$. So, it follows from (21) that

$$
\int_{G} \int_{X}\left|K_{t}(g) \overline{\psi(x)}(U(g) \varphi)(x)\right| d x d g<\infty .
$$

Then Fubini's theorem gives

(23) $(\psi, S, \varphi)$

$$
\begin{aligned}
& =\int_{X} \overline{\psi(\dot{g})} \int_{G} K_{t}(k) S\left(k^{-1} \dot{g}, k\right) R\left(\dot{g}, k^{-1}\right)^{1 / 2} \varphi\left(k^{-1} \dot{g}\right) d k d \dot{g} \\
& =\int_{X} \overline{\psi(\dot{g})} \int_{G} K_{t}\left(k^{-1}\right) S\left(k \dot{g}, k^{-1}\right) R(\dot{g}, k)^{1 / 2} \Delta_{G}\left(k^{-1}\right) \varphi(k \dot{g}) d k d \dot{g} \\
& =\int_{X} \overline{\psi(\dot{g})} \int_{G} K_{t}\left(g k^{-1}\right) S\left(\dot{k}, g k^{-1}\right) R\left(\dot{g}, k g^{-1}\right)^{1 / 2} \Delta_{G}\left(k^{-1}\right) \varphi(\dot{k}) d k d \dot{g} \\
& =\int_{X} \overline{\psi(\dot{g})} \int_{G} K_{t}\left(g k^{-1}\right) S\left(\dot{k}, g k^{-1}\right)(\rho(k) \rho(g))^{-1 / 2} \Delta_{G}\left(k^{-1}\right) \varphi(\dot{k}) \rho(k) d k d \dot{g} .
\end{aligned}
$$

By (7) there exist $C, \eta>0$ such that

$$
(\rho(k) \rho(g))^{-1 / 2}=\rho(e)^{-1} R(\dot{e}, k)^{-1 / 2} R(\dot{e}, g)^{-1 / 2} \leq \rho(e)^{-1} C e^{\eta(k|+| g \mid)},
$$

and $\Delta_{G}\left(k^{-1}\right) \leq C e^{\eta|k|}$ for all $g, k \in G$. Moreover, it follows from (12) that

$$
\left|S\left(\dot{k}, g k^{-1}\right)\right| \leq C^{\prime} e^{\eta^{\prime}(|k|+|g|)}
$$


for all $g, k \in G$. Hence, it follows from (21) that the function

$$
k \mapsto K_{t}\left(g k^{-1}\right) S\left(\dot{k}, g k^{-1}\right)(\rho(k) \rho(g))^{-1 / 2} \Delta_{G}\left(k^{-1}\right) \varphi(\dot{k})
$$

belongs to $L_{1}(G ; \rho d g)$ for all $g \in G$. Then (9) and (23) give

$$
\begin{aligned}
\left(\psi, S_{t} \varphi\right)= & \int_{X} \overline{\psi(\dot{g})} \int_{X}\left(\int_{M} K_{t}\left(g m^{-1} k^{-1}\right) S\left(\dot{k}, g m^{-1} k^{-1}\right)\right. \\
& \left.\times(\rho(g) \rho(k m))^{-1 / 2} \Delta_{G}\left(m^{-1} k^{-1}\right) d m\right) \varphi(\dot{k}) d \dot{k} d \dot{g} \\
= & \int_{X} \overline{\psi(x)} \int_{X} \kappa_{t}(x ; y) \varphi(y) d y d x
\end{aligned}
$$

for all $t>0$ and $\varphi, \psi \in C_{c}(X)$.

Since $C_{c}(X)$ is dense in $L_{p}(X ; d x)$ for all $p \in[1, \infty]$, it follows that (24) is valid for all $\varphi \in L_{p}(X ; d x)$ and $\psi \in L_{q}(X ; d x)$, where $p^{-1}+q^{-1}=1$, and the proof of the proposition is complete.

The function $t \mapsto \kappa_{t}(x ; y)$, with $x, y \in X$ fixed, extends to a holomorphic function, since $S=S_{t}$ is holomorphic (see also [1, Theorem 3.1]).

Now, we discuss the regularity of the reduced heat kernel $\kappa_{t}$. We need some notation. Consider the contragredient

representation of $U$ in each $L_{p}(X ; d x)$ defined by

$$
(\check{U}(g) \varphi)(x)=S\left(x, g^{-1}\right) R\left(x, g^{-1}\right)^{1 / 2} \varphi\left(g^{-1} x\right) \quad(\text { a.e. } x \in X)
$$

for all $\varphi \in L_{p}(X ; d x)$. For all $i \in\{1, \ldots, d\}$, let $R_{i}$ denote the infinitesimal generator in the direction $a_{i}$ affiliated to $\check{U}$. The left derivative in the direction $a_{i}$ on the Lie group $G$ is denoted by $\widetilde{A}_{i}$ and the right derivative by $\widetilde{R}_{i}$. If $\beta \in J(d)$, then by $L b(\beta)$ we denote the set of all $(\gamma, \delta) \in J(d)^{2}$ such that $\gamma$ is a multi-index obtained from $\beta$ by omission of some indices and $\delta$ is the multi-index formed by the omitted indices (see, for example, [4, page 747]). Moreover, if $\delta=\left(j_{1}, \ldots, j_{l}\right) \in J(d)$ then we set $d_{\delta}=\left(\widetilde{R}_{j_{1}} \Delta_{G}\right)(e) \cdots\left(\widetilde{R}_{j_{1}} \Delta_{G}\right)(e)$. Next, we show that, for fixed $t>0$, the heat kernel $\kappa_{t}$ is pointwise $C^{\infty}$ in the second variable, with respect to $R_{1}, \ldots, R_{d}$, and, moreover, if $\beta \in J(d)$ and $R^{\beta}$ denotes the pointwise (multi-)derivative, with respect to the second variable, then $R^{\beta} \kappa_{t}$ is pointwise $C^{\infty}$ in the first variable, with respect to the infinitesimal generators $A_{1}, \ldots, A_{d}$. Further, if $\alpha, \beta \in J(d), A^{\alpha}$ denotes the pointwise (multi-)derivative, with respect to the first variable, and $R^{\beta}$ denotes, again, the pointwise (multi-)derivative, with respect to the second variable, then $A^{\alpha} R^{\beta} K_{1}$ is also given by a reduction formula. The proof is quite similar to the proof of Lemma 2.5 using induction and (22). 
PROPOSITION 2.7. If $t>0$, then the kernel $\kappa_{t}$ is pointwise $C^{\infty}$ in the second variable, with respect to $R_{1}, \ldots, R_{d}$, and if $\beta \in J(d)$, then $R^{\beta} \kappa_{t}$ is pointwise $C^{\infty}$ in the first variable, with respect to $A_{1}, \ldots, A_{d}$. Furthermore, if $\alpha, \beta \in J(d)$ then $A^{\alpha} R^{\beta} \kappa_{t}$ is given by the following reduction formula

$$
\begin{aligned}
\left(A^{\alpha} R^{\beta} \kappa_{t}\right)(\dot{g} ; \dot{k})= & \sum_{(\gamma, \delta) \in L b(\beta)} d_{\delta} \int_{M}\left(\tilde{A}^{\alpha} \widetilde{R}^{\gamma} K_{t}\right)\left(g m^{-1} k^{-1}\right) S\left(\dot{k}, g m^{-1} k^{-1}\right) \\
& \times(\rho(g) \rho(k m))^{-1 / 2} \Delta_{G}\left(m^{-1} k^{-1}\right) d m
\end{aligned}
$$

for all $g, k \in G$.

Next, let $V$ be the representation of $G$ in $L_{\infty}(X ; d x)$ defined by

$$
(V(g) \varphi)(x)=\varphi\left(g^{-1} x\right) \quad(\text { a.e. } x \in X)
$$

for all $\varphi \in L_{\infty}(X ; d x)$ and $g \in G$. Let $B_{i}=d V\left(a_{i}\right)$ denote the infinitesimal generator of the one parameter group $t \mapsto V\left(\exp \left(-t a_{i}\right)\right)$ in $L_{\infty}(X ; d x)$. Consider the metric $d: X \times X \rightarrow[0, \infty)$ on $X$ defined by

$$
d(x ; y)=\sup \left\{|\psi(x)-\psi(y)|: \psi \in C_{b ; \infty}(X) \text { real and } \sum_{i=1}^{d}\left|B_{i} \psi\right|^{2} \leq 1\right\}
$$

for all $x, y \in X$, where $C_{b ; \infty}(X)$ denotes the space of all infinitely differentiable functions on $X$ with uniformly bounded derivatives. Introduce the balls $B(x ; r)=$ $\{y \in X: d(x ; y)<r\}$ for all $x \in X$ and $r>0$.

Now we are able to state the main result of this paper.

THEOREM 2.8. Let $X=G / M$ be a homogeneous space with $G$ a connected Lie group and $M$ a Lie subgroup. Suppose that $R$ is continuous and satisfies (6). Further suppose that $S$ is continuous and satisfies (11). Let $H$ be an $n$-th order strongly elliptic operator associated to $U$, given by (13), and let $\kappa_{t}$ be the corresponding reduced heat kernel. Then for all $\alpha, \beta \in J(d)$ there exist $b, c>0$ and $\omega \geq 0$ such that

$$
\begin{aligned}
\left|\left(A^{\alpha} R^{\beta} \kappa_{t}\right)(x ; y)\right| \leq & c\left(\operatorname{Vol}_{X}(B(x ; 1)) \operatorname{Vol}_{X}(B(y ; 1))\right)^{-1 / 2} t^{-\left(|\alpha|+|\beta|+d-d_{M}\right) / n} e^{\omega t} \\
& \times e^{-b\left(d(x ; y)^{n} t^{-1}\right)^{1 /(n-1)}}
\end{aligned}
$$

for all $x, y \in X$ and $t>0$.

The reduction formulas for the heat kernel and all its derivatives, in the first and second variable, given by (25), are an essential part in the proof of the main Theorem 2.8 given in Section 5. 


\section{A local scaling property of the volume}

We aim to show that condition (6) implies a local scaling property of the volume of the balls $B_{\varepsilon} x$ with $x \in X$ and $\varepsilon \in\langle 0,1]$. More precisely, we deduce the following proposition. See also [16, Chapter 3] and [14].

PROPOSITION 3.1. There exists a $C \geq 1$ such that

$$
\operatorname{Vol}_{X}\left(B_{1} x\right) \leq C \varepsilon^{-\left(d-d_{M}\right)} \operatorname{Vol}_{X}\left(B_{\varepsilon} x\right)
$$

for all $x \in X$ and $\varepsilon \in\langle 0,1]$.

PROOF. Consider the 'spherical' balls

$$
\widetilde{B}_{\varepsilon}=\exp \left(R_{\varepsilon}\right),
$$

where $R_{\varepsilon}=\left\{\sum_{i=1}^{d} t_{i} a_{i} \in \mathfrak{g}: \sum_{i=1}^{d} t_{i}^{2}<\varepsilon^{2}\right\}$ for all $\varepsilon>0$.

The proof is subdivided in 10 steps. First we show that (27) holds if there are $C, N>0$ and $\varepsilon_{0} \in\langle 0,1]$ such that

$$
\operatorname{Vol}_{X}\left(B_{N} x\right) \leq C \varepsilon^{-\left(d-d_{M}\right)} \operatorname{Vol}_{X}\left(B_{\varepsilon} x\right),
$$

uniformly for all $x \in X$ and $\varepsilon \in\left\langle 0, \varepsilon_{0}\right]$. Then we scale the basis $a_{1}, \ldots, a_{d}$ suitably for the sake of convenience, and we reduce the local scaling property of the volume to a uniform estimate of $\operatorname{Vol}_{M}\left(M \cap g^{-1} \widetilde{B}_{\varepsilon} g\right)$ in terms of $\operatorname{Vol}_{M}\left(M \cap g^{-1} \widetilde{B}_{20} g\right)$ for all $\varepsilon \in\left\langle 0, \varepsilon_{0}\right]$, with $\varepsilon_{0} \in\langle 0,1]$, uniformly in $g \in G$. Next, we give a description of the connected components of $M \cap g^{-1} \widetilde{B}_{\varepsilon} g$ for all $\varepsilon \in\langle 0,20]$ and $g \in G$, and we derive a convexity result from it. Then we introduce a metric $\tilde{d}_{G}$ on $g^{-1} \widetilde{B}_{20} g$ and a 'metric' $\tilde{d}_{M}$ on $M \cap g^{-1} \widetilde{B}_{20} g$, and it follows from the convexity result that $\tilde{d}_{G}$ and $\tilde{d}_{M}$ are equivalent metrics on the connected components of $M \cap g^{-1} \widetilde{\boldsymbol{B}}_{\rho} g$, with $\rho \in\langle 0,1]$, uniformly with respect to $g$. Finally, this equivalence implies a homothetic contraction result from which the proposition follows.

Step 1. If there exist $C, N>0$ and $\varepsilon_{0} \in\langle 0,1]$ such that (29) is valid then the proposition follows. This can be proved by the next covering argument. We may assume that $N<1$. There exist $L \in \mathbb{N}$ and $g_{1}, \ldots, g_{L} \in B_{1}$ such that $B_{1} \subseteq$ $\bigcup_{i=1}^{L} g_{i} B_{N}$. So, $B_{1} x \subseteq \bigcup_{i=1}^{L} g_{i} B_{N} x$ for all $x \in X$. Next, for all $i \in\{1, \ldots, L\}$ and $x \in X$, one has

$$
\operatorname{Vol}_{X}\left(g_{i} B_{N} x\right)=\int_{X} 1_{B_{N} x}\left(g_{i}^{-1} y\right) d y=\int_{X} 1_{B_{N} x}(y) R\left(y, g_{i}\right) d y \leq C_{R} \operatorname{Vol}_{X}\left(B_{N} x\right) .
$$

Therefore, (29) implies that

$$
\operatorname{Vol}_{X}\left(B_{1} x\right) \leq L C_{R} \operatorname{Vol}_{X}\left(B_{N} x\right) \leq L C C_{R} \varepsilon^{-\left(d-d_{M}\right)} \operatorname{Vol}_{X}\left(B_{\varepsilon} x\right)
$$


for all $\varepsilon \in\left\langle 0, \varepsilon_{0}\right]$ and $x \in X$. But then it is obvious that

$$
\operatorname{Vol}_{X}\left(B_{1} x\right) \leq L C C_{R} \varepsilon_{0}^{-\left(d-d_{M}\right)} \operatorname{Vol}_{X}\left(B_{\varepsilon_{0}} x\right) \leq L C C_{R} \varepsilon_{0}^{-\left(d-d_{M}\right)} \operatorname{Vol}_{X}\left(B_{\varepsilon} x\right) \varepsilon^{-\left(d-d_{M}\right)},
$$

uniformly for all $x \in X$ and $\varepsilon \in\left\langle\varepsilon_{0}, 1\right]$, and (27) holds.

Step 2. In this step we scale the basis $a_{1}, \ldots, a_{d}$ for the sake of convenience.

Without loss of generality, we may assume the following.

- The Baker-Campbell-Hausdorff formula is valid (convergent) on $R_{200}$, the exponential map is a real analytic diffeomorphism from $R_{600}$ onto $\widetilde{B}_{600}$ and

$$
\widetilde{B}_{\varepsilon} \widetilde{B}_{\varepsilon} \subseteq \widetilde{B}_{3 \varepsilon}
$$

for all $\varepsilon \in\langle 0,200]$.

- By the inverse function theorem, there exist $\xi \geq 1$ and $\nu \geq 1$ such that for all $l, k \in\{1, \ldots, d\}$ there exists a function $\alpha_{l k}: \mathbb{R}^{d} \rightarrow \mathbb{R}$ such that

$$
\begin{aligned}
& \exp \left(\sum_{i=1}^{d} s_{i} a_{i}\right) \exp \left(\sum_{i=1}^{d} t_{i} a_{i}\right) \\
& =\exp \left(\sum_{i=1}^{d}\left(s_{i}+t_{i}\right) a_{i}+\sum_{l=1}^{d}\left(\sum_{k=1}^{d} t_{k} \alpha_{l k}(s)\right) a_{l}+O\left(|t|^{2}\right)\right)
\end{aligned}
$$

for all $s, t \in \mathbb{R}^{d}$ such that $|s|,|t|<200$, and, moreover, $\left\|(I+A(s))^{-1}\right\| \leq \xi$ and $\|I+A(s)\| \leq \xi$ for all $s \in \mathbb{R}^{d}$ with $|s|<200$, where $A(s) \in \mathbb{R}^{d \times d}$ is the matrix given by $(A(s))_{l k}=\alpha_{l k}(s)$ for all $l, k \in\{1, \ldots, d\}$, and for all $U \in S O(d, \mathbb{R})$

$$
\nu^{-1} \leq \operatorname{det}\left(I+A_{U}(s)\right) \leq \nu, \quad\left\|\left(I+A_{U}(s)\right)^{-1}\right\| \leq \xi \quad \text { and } \quad\left\|I+A_{U}(s)\right\| \leq \xi
$$

for all $s \in \mathbb{R}^{d}$ with $|s|<200$, where $A_{U}(s) \in \mathbb{R}^{d_{M} \times d_{M}}$ is the matrix given by $\left(A_{U}(s)\right)_{l k}=\left(U A(s) U^{-1}\right)_{l k}$ for all $l, k \in\left\{1, \ldots, d_{M}\right\}$.

- There is a $K \geq 1$ such that

$$
\widetilde{B}_{K^{-1} \varepsilon} \subseteq B_{2 \varepsilon} \subseteq \widetilde{B}_{K \varepsilon}
$$

for all $\varepsilon \in\langle 0,200 K]$ (see also [5, Proposition 6.1]).

Next, fix $g \in G$. Then $g^{-1} \widetilde{B}_{\varepsilon} g=\exp \left(P_{\varepsilon}^{g}\right)$, where

$$
P_{\varepsilon}^{g}=\left\{\sum_{i=1}^{d} t_{i} \hat{a}_{i} \in \mathfrak{g}: \sum_{i=1}^{d} t_{i}^{2}<\varepsilon^{2}\right\},
$$

for all $\varepsilon>0$, where $\hat{a}_{i}=\operatorname{Ad}\left(g^{-1}\right) a_{i}$ for all $i \in\{1, \ldots, d\}$. Endow $g$ with the inner product $\left(\sum_{i=1}^{d} t_{i} \hat{a}_{i}, \sum_{i=1}^{d} s_{i} \hat{a}_{i}\right)=\sum_{i=1}^{d} t_{i} s_{i}$ for all $\sum_{i=1}^{d} t_{i} \hat{a}_{i} \in \mathfrak{g}$ and $\sum_{i=1}^{d} s_{i} \hat{a}_{i} \in \mathfrak{g}$. 
Then there is an orthonormal transformation $T \in S O(\mathfrak{g})$ such that $\tilde{a}_{i}=T\left(\hat{a}_{i}\right)$, $i \in\{1, \ldots, d\}$, establishes an orthonormal basis of $\mathfrak{g}$, and $\tilde{a}_{1}, \ldots, \tilde{a}_{d_{M}}$ is a basis of $\mathfrak{m}$. Then $P_{\varepsilon}^{g}=\left\{\sum_{i=1}^{d} u_{i} \tilde{a}_{i} \in \mathfrak{g}: \sum_{i=1}^{d} u_{i}^{2}<\varepsilon^{2}\right\}$. So, $P_{\varepsilon}^{g}$ is a 'spherical' ball in $\mathfrak{g}$ with center 0 and radius $\varepsilon$ for all $\varepsilon>0$. By (31) and (32) the following two statements are valid.

- For all $l, k \in\{1, \ldots, d\}$ there exists a function $\rho_{l k}: \mathbb{R}^{d} \rightarrow \mathbb{R}$ such that

$$
\begin{aligned}
\exp \left(\sum_{i=1}^{d} s_{i} \tilde{a}_{i}\right) \exp \left(\sum_{i=1}^{d} t_{i} \tilde{a}_{i}\right) \\
=g^{-1} \exp \left(\sum_{i=1}^{d} s_{i} \operatorname{Ad}(g) \tilde{a}_{i}\right) \exp \left(\sum_{i=1}^{d} t_{i} \operatorname{Ad}(g) \tilde{a}_{i}\right) g \\
=g^{-1} \exp \left(\sum_{i=1}^{d}\left(s_{i}+t_{i}\right) \operatorname{Ad}(g) \tilde{a}_{i}\right. \\
\left.\quad+\sum_{l=1}^{d}\left(\sum_{k=1}^{d} t_{k} \rho_{l k}(s)\right) \operatorname{Ad}(g) \tilde{a}_{l}+O\left(|t|^{2}\right)\right) g \\
=\exp \left(\sum_{i=1}^{d}\left(s_{i}+t_{i}\right) \tilde{a}_{i}+\sum_{l=1}^{d}\left(\sum_{k=1}^{d} t_{k} \rho_{l k}(s)\right) \tilde{a}_{l}+O\left(|t|^{2}\right)\right)
\end{aligned}
$$

for all $s, t \in \mathbb{R}^{d}$ such that $|s|,|t|<200$, and, moreover, $\left\|(I+R(s))^{-1}\right\| \leq \xi$ and $\|I+R(s)\| \leq \xi$ for all $s \in \mathbb{R}^{d}$ with $|s|<200$, where $R(s) \in \mathbb{R}^{d \times d}$ denotes the matrix given by $(R(s))_{l k}=\rho_{l k}(s)$ for all $l, k \in\{1, \ldots, d\}$.

- Similarly, for all $l, k \in\left\{1, \ldots, d_{M}\right\}$ there exists a function $\omega_{l k}: \mathbb{R}^{d_{M}} \rightarrow \mathbb{R}$ such that

$$
\begin{aligned}
& \exp \left(\sum_{i=1}^{d_{M}} s_{i} \tilde{a}_{i}\right) \exp \left(\sum_{i=1}^{d_{M}} t_{i} \tilde{a}_{i}\right) \\
& \quad=\exp \left(\sum_{i=1}^{d_{M}}\left(s_{i}+t_{i}\right) \tilde{a}_{i}+\sum_{l=1}^{d_{M}}\left(\sum_{k=1}^{d_{M}} t_{k} \omega_{l k}(s)\right) \tilde{a}_{l}+O\left(|t|^{2}\right)\right)
\end{aligned}
$$

for all $s, t \in \mathbb{R}^{d_{M}}$ such that $|s|,|t|<200$, and, moreover, $\left\|(I+\Omega(s))^{-1}\right\| \leq \xi$ and $\|I+\Omega(s)\| \leq \xi$ for all $s \in \mathbb{R}^{d_{M}}$ with $|s|<200$, and

$$
v^{-1} \leq \operatorname{det}(I+\Omega(s)) \leq v
$$

for all $s \in \mathbb{R}^{d_{M}}$ with $|s|<200$, where $\Omega(s) \in \mathbb{R}^{d_{M} \times d_{M}}$ denotes the matrix given by $(\Omega(s))_{l k}=\omega_{l k}(s)$ for all $l, k \in\left\{1, \ldots, d_{M}\right\}$. 
Step 3. In this step we show that

$$
\operatorname{Vol}_{X}\left(B_{\varepsilon} \dot{g}\right) \geq C_{R}^{-1} \Delta_{G}(g) \rho(g) \operatorname{Vol}_{G}\left(B_{\varepsilon}\right)\left(\int_{M \cap g^{-1} B_{2 \varepsilon} g} 1 d m\right)^{-1}
$$

for all $\varepsilon \in\langle 0,1]$, and

$$
\operatorname{Vol}_{X}\left(B_{\varepsilon} \dot{g}\right) \leq C_{R}^{3 \varepsilon+2} \Delta_{G}(g) \rho(g) \operatorname{Vol}_{G}\left(B_{3 \varepsilon}\right)\left(\int_{M \cap g^{-1} B_{2 \varepsilon} g} 1 d m\right)^{-1}
$$

for all $\varepsilon>0$.

First we deduce (37). Let $\varepsilon \in\langle 0,1]$. Note first that $h \mapsto 1_{B_{\varepsilon}}\left(h g^{-1}\right) \rho(h)^{-1}$ belongs to $L_{1}(G ; \rho d g)$, because the support is compact. Then

$$
\begin{aligned}
\operatorname{Vol}_{G}\left(B_{\varepsilon}\right) & =\int_{G} 1_{B_{\varepsilon}}\left(h g^{-1}\right) \Delta_{G}\left(g^{-1}\right) d h \\
& =\int_{X} \int_{M} 1_{B_{\varepsilon}}\left(h m g^{-1}\right) \Delta_{G}\left(g^{-1}\right) \rho(h m)^{-1} d m d \dot{h}
\end{aligned}
$$

by Lemma 2.2 (II).

Next, if $h \in G, m \in M$ and $h m g^{-1} \in B_{\varepsilon}$ then there exists a $b \in B_{\varepsilon}$ such that $h m=b g$. Hence,

$$
\rho(h m)^{-1}=\rho(b g)^{-1}=\rho(g)^{-1} R(\dot{g}, b)^{-1} \leq C_{R} \rho(g)^{-1}
$$

by Lemma 2.2 (III). Therefore,

$$
\int_{M} 1_{B_{\varepsilon}}\left(h m g^{-1}\right) \Delta_{G}\left(g^{-1}\right) \rho(h m)^{-1} d m \leq C_{R} \Delta_{G}\left(g^{-1}\right) \rho(g)^{-1} \int_{M} 1_{B_{\varepsilon}}\left(h m g^{-1}\right) d m
$$

for all $h \in G$. Moreover, if $h \in G$ and there exists an $m_{1} \in M$ such that $h m_{1} g^{-1} \in B_{\varepsilon}$ then $h m_{1} \in B_{\varepsilon} g$, and thus $m_{1}^{-1} h^{-1} \in g^{-1} B_{\varepsilon}$. Hence, $m_{1}^{-1} h^{-1} B_{\varepsilon} g \subseteq g^{-1} B_{2 \varepsilon} g$. If $m \in M$ and $h m_{1} m g^{-1} \in B_{\varepsilon}$ then $m \in M \cap m_{1}^{-1} h^{-1} B_{\varepsilon} g \subseteq M \cap g^{-1} B_{2 \varepsilon} g$. Therefore, since $d m$ is left invariant, one has

$$
\int_{M} 1_{B_{i}}\left(h m g^{-1}\right) d m=\int_{M} 1_{B_{\varepsilon}}\left(h m_{1} m g^{-1}\right) d m \leq \int_{M \cap g^{-1} B_{2} g} 1 d m .
$$

It follows that $\int_{M} 1_{B_{t}}\left(h m g^{-1}\right) d m \leq \int_{M \cap g^{-1} B_{2+} g} 1 d m$ for all $h \in G$. So,

$$
\int_{M} 1_{B_{i}}\left(h m g^{-1}\right) \Delta_{G}\left(g^{-1}\right) \rho(h m)^{-1} d m \leq C_{R} \Delta_{G}\left(g^{-1}\right) \rho(g)^{-1} \int_{M \cap g^{-1} B_{x} g} 1 d m
$$

for all $h \in G$. If $h \in G$ is such that $\int_{M} 1_{B_{i}}\left(h m g^{-1}\right) \Delta_{G}\left(g^{-1}\right) \rho(h m)^{-1} d m \neq 0$, then there exists an $m \in M$ such that $h m g^{-1} \in B_{\varepsilon}$. Hence, $\dot{h} \in B_{\varepsilon} \dot{g}$. Therefore,

$$
\operatorname{Vol}_{G}\left(B_{\varepsilon}\right) \leq C_{R} \Delta_{G}\left(g^{-1}\right) \rho(g)^{-1} \int_{B_{\dot{\alpha}}} \int_{M \cap g^{-1} B_{2, g}} 1 d m d \dot{h}
$$




$$
=C_{R} \Delta_{G}\left(g^{-1}\right) \rho(g)^{-1} \operatorname{Vol}_{X}\left(B_{\varepsilon} \dot{g}\right) \int_{M \cap g^{-1} B_{2 \varepsilon} g} 1 d m
$$

for all $\varepsilon \in(0,1]$, and (37) follows immediately.

Next, we derive (38). Let $\varepsilon>0$. If $h \in B_{\varepsilon} g$, then

$$
\rho(h m) \rho(g m)^{-1}=\rho(h) \rho(g)^{-1}=R\left(\dot{g}, h g^{-1}\right) \leq C_{R}^{\varepsilon+1}
$$

for all $m \in M$. If, in addition, $m \in g^{-1} B_{2 \varepsilon} g$, then $h m \in B_{3 \varepsilon} g$. Hence,

$$
\begin{aligned}
\int_{M} 1_{g^{-1} B_{2 \epsilon} g}(m) \rho(g m)^{-1} d m & \leq C_{R}^{\varepsilon+1} \int_{M} 1_{g^{-1} B_{2 \epsilon} g}(m) \rho(h m)^{-1} d m \\
& \leq C_{R}^{\varepsilon+1} \int_{M} 1_{B_{3 \varepsilon} g}(h m) \rho(h m)^{-1} d m
\end{aligned}
$$

for all $h \in B_{\varepsilon} g$. Then

$$
1_{B_{\varepsilon} \dot{g}}(\dot{h}) \int_{M} 1_{g^{-1} B_{2 \varepsilon} g}(m) \rho(g m)^{-1} d m \leq C_{R}^{\varepsilon+1} \int_{M} 1_{B_{3 \varepsilon} g}(h m) \rho(h m)^{-1} d m
$$

for all $h \in G$, since $h \mapsto \int_{M} 1_{B_{3_{k}} g}(h m) \rho(h m)^{-1} d m$ is right $M$-invariant.

Finally, note that $h \mapsto 1_{B_{3 \varepsilon} g}(h) \rho(h)^{-1}$ belongs to $L_{1}(G ; \rho d g)$, because the support is compact. So, integration over $X$ yields

$$
\text { (41) } \begin{aligned}
\operatorname{Vol}_{X}\left(B_{\varepsilon} \dot{g}\right) \int_{M \cap g^{-1} B_{2 \varepsilon} g} \rho(g m)^{-1} d m & \leq C_{R}^{\varepsilon+1} \int_{X}\left(\int_{M} 1_{B_{3_{\varepsilon}} g}(h m) \rho(h m)^{-1} d m\right) d \dot{h} \\
& =C_{R}^{\varepsilon+1} \int_{G} 1_{B_{3 \varepsilon} g}(h) d h \\
& =C_{R}^{\varepsilon+1} \operatorname{Vol}_{G}\left(B_{3 \varepsilon}\right) \Delta_{G}(g),
\end{aligned}
$$

by the Weyl formula. But, if $m \in M \cap g^{-1} B_{2 \varepsilon} g$, then there is a $b \in B_{2 \varepsilon}$ such that $g m=b g$. Hence one obtains

$$
\rho(g m)^{-1}=\rho(b g)^{-1} \geq C_{R}^{-2 \varepsilon-1} \rho(g)^{-1},
$$

and (38) follows immediately from (41) and (42).

Step 4. Let $N=20 K$. It follows from (37) and (38) that

$$
\begin{aligned}
\operatorname{Vol}_{X}\left(B_{N} \dot{g}\right) & \leq C_{R}^{60 K+2} \Delta_{G}(g) \rho(g) \frac{\operatorname{Vol}_{G}\left(B_{3 N}\right)}{\int_{M \cap g^{-1} B_{2 N} g} 1 d m} \\
& \leq C_{R}^{63 K} \operatorname{Vol}_{G}\left(B_{3 N}\right) \operatorname{Vol}_{G}\left(B_{\varepsilon}\right)^{-1} \int_{M \cap g^{-1} B_{2+} g} 1 d m \frac{\operatorname{Vol}_{X}\left(B_{\varepsilon} \dot{g}\right)}{\int_{M \cap g^{-1} B_{40} K g} 1 d m}
\end{aligned}
$$

for all $\varepsilon \in\langle 0,1]$. There is a $C \geq 1$ such that

$$
\operatorname{Vol}_{G}\left(B_{3 N}\right) \operatorname{Vol}_{G}\left(B_{\varepsilon}\right)^{-1} \leq C \varepsilon^{-d}
$$


for all $\varepsilon \in\langle 0,1]$. Therefore, the proof of the proposition is complete if there exist $C \geq 1$ and $\varepsilon_{0} \in\{0,1]$, independent of $g$, such that

$$
\operatorname{Vol}_{M}\left(M \cap g^{-1} \widetilde{B}_{\varepsilon} g\right) \leq C \varepsilon^{d_{M}} \operatorname{Vol}_{M}\left(M \cap g^{-1} \widetilde{B}_{20} g\right)
$$

for all $\varepsilon \in\left\langle 0, \varepsilon_{0}\right]$. Indeed, if (43) is valid then it follows from (33) that

$$
\mathrm{Vol}_{M}\left(M \cap g^{-1} B_{2 \varepsilon} g\right) \leq C K^{d_{M}} \varepsilon^{d_{M}} \operatorname{Vol}_{M}\left(M \cap g^{-1} B_{40 K} g\right)
$$

for all $\varepsilon \in\left\langle 0, K^{-1} \varepsilon_{0}\right]$. It follows that there is a $C^{\prime} \geq 1$, independent of $g$, such that

$$
\operatorname{Vol}_{X}\left(B_{N} \dot{g}\right) \leq C^{\prime} \varepsilon^{-\left(d-d_{M}\right)} \operatorname{Vol}_{X}\left(B_{\varepsilon} \dot{g}\right)
$$

for all $\varepsilon \in\left\langle 0, K^{-1} \varepsilon_{0}\right]$, and (29) holds.

Step 5. If $\varepsilon>0$ is small then it follows from [6, Lemma 2.2] that

$$
M \cap \widetilde{B}_{\varepsilon}=\exp \left(\left\{\sum_{i=1}^{d_{M}} u_{i} a_{i} \in \mathfrak{m}: \sum_{i=1}^{d_{M}} u_{i}^{2}<\varepsilon^{2}\right\}\right) .
$$

Unfortunately, the sets $M \cap g^{-1} \widetilde{B}_{\varepsilon} g$ are not uniformly bounded with respect to $g$. Nevertheless, an exponential description of the above kind is still valid with respect to the basis $\tilde{a}_{1}, \ldots, \tilde{a}_{d_{M}}$ for $\mathrm{m}$. On connected subsets the description is possible uniformly in $g$ and $\varepsilon \in\langle 0,20]$.

LEMMA 3.2. If $\varepsilon \in\langle 0,20], \mathscr{C}$ is a connected component of the set $M \cap g^{-1} \widetilde{B}_{\varepsilon} g$ and $m_{0} \in \mathscr{C}$, then for each $m \in \mathscr{C}$ there is a $u \in \mathbb{R}^{d_{M}}$ such that $m=m_{0} \exp \left(\sum_{i=1}^{d_{M}} u_{i} \tilde{a}_{i}\right)$ and $|u|<3 \varepsilon$. In particular, there is a non-empty open connected neighbourhood $O \subseteq \mathfrak{m} \cap P_{3 \varepsilon}^{g}$ of 0 such that $\mathscr{C}=m_{0} \exp (O)$.

PROOF. Let $m \in \mathscr{C}$. Since there is an absolutely continuous path from $m_{0}$ to $m$ in $\mathscr{C}$, there are $n \in \mathbb{N}$ and a sequence of points $m_{0}, m_{1}, \ldots, m_{n} \in \mathscr{C}$ such that $m_{n}=m, g m_{0} g^{-1}, \ldots, g m_{n} g^{-1} \in \widetilde{B}_{\varepsilon}$, and for all $k \in\{1, \ldots, n\}$ there exists a $u \in \mathbb{R}^{d_{M}}$ such that $m_{k-1}^{-1} m_{k}=\exp \left(\sum_{i=1}^{d_{M}} u_{i} \tilde{a}_{i}\right)$ and $|u|<200$. But then $|u|<3 \varepsilon$, because $m_{k-1}^{-1} m_{k} \in g^{-1} \widetilde{B}_{3 \varepsilon} g$ for all $k \in\{1, \ldots, n\}$, by (30).

Next, $m_{0}=m_{0} \exp (0) \in g^{-1} \widetilde{B}_{\varepsilon} g$. Let $0 \leq k<n$, and suppose that there is a $v \in \mathbb{R}^{d_{M}}$ such that $m_{k}=m_{0} \exp \left(\sum_{i=1}^{d_{M}} v_{i} \tilde{a}_{i}\right)$ and $|v|<3 \varepsilon$. Since $m_{k}^{-1} m_{k+1} \in g^{-1} \widetilde{B}_{3 \varepsilon} g$ and the Baker-Campbell-Hausdorff formula is valid on $R_{3 \varepsilon}$, there is a $v \in \mathbb{R}^{d_{M}}$ such that $m_{k+1}=m_{k} m_{k}^{-1} m_{k+1}=m_{0} \exp \left(\sum_{i=1}^{d_{M}} v_{i} \tilde{a}_{i}\right)$ and $|v|<9 \varepsilon$ by (30). As $m_{k+1}, m_{0}^{-1} \in g^{-1} \widetilde{B}_{\varepsilon} g$, it follows that $m_{0}^{-1} m_{k+1}=\exp \left(\sum_{i=1}^{d_{M}} v_{i} \tilde{a}_{i}\right) \in g^{-1} \widetilde{B}_{3 \varepsilon} g$, by (30) again. Hence, $m_{k+1}=m_{0} \exp \left(\sum_{i=1}^{d_{M}} v_{i} \tilde{a}_{i}\right)$, with $|v|<3 \varepsilon$.

By induction, there is a $u \in \mathbb{R}^{d_{M}}$ such that $m=m_{n}=m_{0} \exp \left(\sum_{i=1}^{d_{M}} u_{i} \tilde{a}_{i}\right)$ and $|u|<3 \varepsilon$, as was to be shown. 
Step 6. In this step our goal is to derive the following convexity result.

LEMMA 3.3. If $\mathscr{C}$ is a connected component of $M \cap g^{-1} \widetilde{B}_{20} g, \varepsilon \in\langle 0,1]$ and $m_{0} \in \mathscr{C} \cap g^{-1} \widetilde{B}_{\varepsilon} g$, then there exists an open connected and convex neighbourhood $O \subseteq \mathfrak{m} \cap P_{3 \varepsilon}^{g}$ of 0 such that $\mathscr{C} \cap g^{-1} \widetilde{B}_{\varepsilon} g \subseteq m_{0} \exp (O) \subseteq \overline{m_{0} \exp (O)} \subseteq \mathscr{C}$.

ProOF. There exists a countable index set $I$ such that the $\mathscr{C}_{\alpha}$, with $\alpha \in I$, are the connected components of $\mathscr{C} \cap g^{-1} \widetilde{\boldsymbol{B}}_{\varepsilon} g$. Since $m_{0} \in \mathscr{C} \cap g^{-1} \widetilde{\boldsymbol{B}}_{\varepsilon} g$ and $\mathscr{C}$ is a connected component of $M \cap g^{-1} \widetilde{B}_{20} g$, it follows from Lemma 3.2 that there exist open connected sets $O_{\alpha} \subseteq \mathfrak{m} \cap P_{200}^{g}$ such that $\mathscr{C}_{\alpha}=m_{0} \exp \left(O_{\alpha}\right)$ for all $\alpha \in I$. Let $\alpha \in I$ and fix $\sum_{i=1}^{d_{M}} u_{i} \tilde{a}_{i} \in O_{\alpha}$. Then there is an $m \in \mathscr{C}_{\alpha}$ such that $m=m_{0} \exp \left(\sum_{i=1}^{d_{M}} u_{i} \tilde{a}_{i}\right)$. Since $m_{0}^{-1}, m \in M \cap g^{-1} \widetilde{B}_{\varepsilon} g$, it follows that

$$
\exp \left(\sum_{i=1}^{d_{M}} u_{i} \tilde{a}_{i}\right)=m_{0}^{-1} m \in M \cap g^{-1} \widetilde{B}_{3 \varepsilon} g=\exp \left(\mathfrak{m} \cap P_{3 \varepsilon}^{g}\right),
$$

by (30). Therefore, $\sum_{i=1}^{d_{M}} u_{i} \tilde{a}_{i} \in \mathfrak{m} \cap P_{3 \varepsilon}^{g}$. So, $O_{\alpha} \subseteq \mathfrak{m} \cap P_{3 \varepsilon}^{g}$ for all $\alpha \in l$.

Next, let $N \in \mathbb{N}, \alpha_{1}, \ldots, \alpha_{N} \in I$ and fix $m_{i} \in \mathscr{C}_{\alpha_{i}}$ for all $i \in\{1, \ldots, N\}$. Then there exist $u^{(i)} \in \mathbb{R}^{d_{M}}$ such that $m_{i}=m_{0} \exp \left(\sum_{k=1}^{d_{M}} u_{k}^{(i)} \tilde{a}_{k}\right)$ and, moreover, $\left|u^{(i)}\right|<3 \varepsilon$ for all $i \in\{1, \ldots, N\}$. Therefore, if $\sum_{i=1}^{N} t_{i}=1$ with $t_{1}, \ldots, t_{N} \geqq 0$, then the convexity of the ball $\widetilde{B}_{3 \varepsilon}$ implies that $\exp \left(\sum_{k=1}^{d_{M}}\left(\sum_{i=1}^{N} t_{i} u_{k}^{(i)}\right) \tilde{a}_{k}\right) \in g^{-1} \widetilde{\widetilde{B}}_{3 \varepsilon} g$. Moreover,

$$
m_{0} \exp \left(\sum_{k=1}^{d_{M}}\left(\sum_{i=1}^{N} t_{i} u_{k}^{(i)}\right) \tilde{a}_{k}\right) \in M \cap g^{-1} \widetilde{B}_{9_{\varepsilon}} g \subseteq M \cap g^{-1} \widetilde{B}_{20} g,
$$

by (30). Let $O \subseteq \mathfrak{m} \cap P_{3 \varepsilon}^{g}$ be the convex hull of the sets $\left\{O_{\alpha}: \alpha \in I\right\}$. Then $O$ is a convex open and connected neighbourhood of 0 . There is a $\delta>0$ such that $\widetilde{O}=(1+\delta) O$ is a convex open and connected neighbourhood of 0 , and, $m_{0} \exp (\widetilde{O}) \subseteq M \cap g^{-1} \widetilde{B}_{10} g$. Since $m_{0} \exp (\widetilde{O})$ is connected and contains $m_{0}$, the lemma follows immediately.

Step 7. In this step we introduce a metric $\tilde{d}_{G}$ on $g^{-1} \widetilde{B}_{20} g$, and we derive an equivalence result.

Consider the map $\Phi: \mathbb{R}^{d} \rightarrow G$ defined by $\Phi(u)=\exp \left(\sum_{i=1}^{d_{M}} u_{i} \tilde{a}_{i}\right), u \in \mathbb{R}^{d}$. Note that $\Phi$ is a diffeomorphism from $\widetilde{N}$ onto $\Phi(\tilde{N})$, where $\widetilde{N}=\left\{u \in \mathbb{R}^{d}:|u|<200\right\}$. For all $i \in\{1, \ldots, d\}$, introduce the right derivatives by

$$
\left(\hat{R}_{i} \varphi\right)(h)=\left.\frac{d}{d t}\right|_{t=0} \varphi\left(h \exp \left(t \tilde{a}_{i}\right)\right)
$$

for all $\varphi \in C_{c}^{\infty}(G)$. 
LEMMA 3.4. The following two statements are valid:

(I) For all $i, k \in\{1, \ldots, d\}$, there exists a function $b_{i k}: g^{-1} \widetilde{B}_{20} g \rightarrow \mathbb{B}$ such that

$$
\left.\hat{R}_{i}\right|_{h}=\left.\sum_{k=1}^{d} b_{i k}(h) d \Phi\left(\frac{\partial}{\partial u_{k}}\right)\right|_{h}
$$

and $\|B(h)\| \leq \xi$ for all $h \in g^{-1} \widetilde{B}_{20} g$, where $B(h) \in \mathbb{R}^{d \times d}$ denotes the matrix given by $(B(h))_{i k}=b_{i k}(h)$.

(II) For all $i, k \in\{1, \ldots, d\}$, there exists a function $c_{i k}: g^{-1} \widetilde{B}_{20} g \rightarrow \mathbb{R}$ such that

$$
\left.d \Phi\left(\frac{\partial}{\partial u_{i}}\right)\right|_{h}=\left.\sum_{k=1}^{d} c_{i k}(h) \hat{R}_{k}\right|_{h}
$$

and $\|C(h)\| \leq \xi$ for all $h \in g^{-1} \widetilde{B}_{20} g$, where $C(h) \in \mathbb{R}^{d \times d}$ is the matrix given by $(C(h))_{i k}=c_{i k}(h)$.

PROOF. It follows from (34), that there is a $t_{0}>0$, independent of $g$, such that for all $i, k \in\{1, \ldots, d\}$ there is a function $\rho_{i k}: \mathbb{R}^{d} \rightarrow \mathbb{R}$ such that

$$
h \exp \left(t \tilde{a}_{i}\right)=\exp \left(\sum_{k=1}^{d} u_{k} \tilde{a}_{k}+t \tilde{a}_{i}+t \sum_{k=1}^{d} \rho_{i k}(u) \tilde{a}_{k}+O\left(|t|^{2}\right)\right)
$$

for all $t \in\left\langle-t_{0}, t_{0}\right)$ and $h=\exp \left(\sum_{k=1}^{d} u_{k} \tilde{a}_{k}\right)$ with $|u|<20$ and, moreover,

$$
\|I+\widetilde{R}(u)\| \leq \xi \quad \text { and } \quad\left\|(I+\widetilde{R}(u))^{-1}\right\| \leq \xi,
$$

where $\widetilde{R}(u) \in \mathbb{R}^{d \times d}$ is the matrix defined by $(\widetilde{R}(u))_{i k}=\rho_{i k}(u)$ for all $i, k \in\{1, \ldots, d\}$. Let $h \in g^{-1} \widetilde{B}_{20} g$. For all $i, k \in\{1, \ldots, d\}$ define the function $r_{i k}: g^{-1} \widetilde{B}_{20} g \rightarrow \mathbb{R}$ by $r_{i k}(h)=\rho_{i k}(u)$ for all $h=\left(\sum_{k=1}^{d} u_{k} \tilde{a}_{k}\right)$ with $|u|<20$. Then

$$
h \exp \left(t \tilde{a}_{i}\right)=\exp \left(\sum_{k=1}^{d} u_{k} \tilde{a}_{k}+t \tilde{a}_{i}+t \sum_{k=1}^{d} r_{i k}(h) \tilde{a}_{k}+O\left(|t|^{2}\right)\right)
$$

for all $t \in\left\langle-t_{0}, t_{0}\right\rangle$ and $h=\exp \left(\sum_{k=1}^{d} u_{k} \tilde{a}_{k}\right)$ with $|u|<20$ and, moreover, $\|B(h)\| \leq \xi$, where $B(h) \in \mathbb{R}^{d \times d}$ is the matrix defined by $(B(h))_{i k}=b_{i k}(h)=\delta_{i k}+r_{i k}(h)$ for all $i, k \in\{1, \ldots, d\}$. Hence, (44) holds for all $h \in g^{-1} \widetilde{B}_{20} g$ and $i \in\{1, \ldots, d\}$, and Statement (I) follows.

If $C(h)=(B(h))^{-1}$ for all $h \in g^{-1} \widetilde{B}_{20} g$ then $\|C(h)\|=\left\|(B(h))^{-1}\right\| \leq \xi$ and, moreover, (45) holds for all $h \in g^{-1} \widetilde{B}_{20} g$, where $c_{i k}(h)=(C(h))_{i k}$ for all $i, k \in$ $\{1, \ldots, d\}$, and Statement (II) follows at once. The proof is complete. 
Let $\tilde{d}_{G}: g^{-1} \widetilde{B}_{20} g \times g^{-1} \widetilde{\boldsymbol{B}}_{20} g \rightarrow[0, \infty\rangle$ denote the metric defined by

$$
\tilde{d}_{G}(h, k)=\inf \left\{\delta>0: \Gamma_{\delta}(h, k) \neq \emptyset\right\},
$$

where $\Gamma_{\delta}(h, k)$ denotes the set of all absolutely continuous paths $\gamma:[0,1] \rightarrow g^{-1} \widetilde{B}_{20} g$ such that $\gamma(0)=h, \gamma(1)=k, \dot{\gamma}(t)=\left.\sum_{i=1}^{d} \gamma_{i}(t) \hat{R}_{i}\right|_{\gamma(t)}$ and $\sum_{i=1}^{d}\left|\gamma_{i}(t)\right|^{2}<\delta^{2}$ for a.e. $t \in[0,1]$.

COROLlaRY 3.5. (I) The metrics $|\cdot|$ and $\tilde{d}_{G}$ satisfy the following equivalence:

$$
\xi^{-1}|u-v| \leq \tilde{d}_{G}(\Phi(u), \Phi(v)) \leq \xi|u-v|
$$

for all $u, v \in \mathbb{R}^{d}$ such that $|u|,|v|<20$.

(II) There is a $\rho \in\left\langle 0, K^{-1}\right]$, independent of $g$, such that

$$
\tilde{d}_{G}(\Phi(u), \Phi(v))=\tilde{d}_{G}(\Phi(w) \Phi(u), \Phi(w) \Phi(v))
$$

for all $u, v, w \in \mathbb{R}^{d}$ such that $|u|,|v|,|w|<\rho$.

PRoOF. Let $u, v \in \mathbb{R}^{d}$ be such that $|u|,|v|<20$. Moreover, let $\gamma:[0,1] \rightarrow$ $g^{-1} \widetilde{B}_{20} g$ be the absolutely continuous path defined by $\gamma(t)=\Phi(t v+(1-t) u)$ for all $t \in[0,1]$. Then $\gamma(0)=\Phi(u), \gamma(1)=\Phi(v)$, and the path lies entirely in $g^{-1} \widetilde{B}_{20} g$, because $P_{20}^{g}$ is a convex set. Moreover,

$$
\dot{\gamma}(t)=\left.\sum_{k=1}^{d} \gamma_{k}(t) d \Phi\left(\frac{\partial}{\partial u_{k}}\right)\right|_{\gamma(t)},
$$

and $\sum_{k=1}^{d}\left|\gamma_{k}(t)\right|^{2}=|u-v|^{2}$ for all $t \in[0,1]$. Hence, it follows from Lemma 3.4 (II) that one can write $\dot{\gamma}(t)=\left.\sum_{k=1}^{d} \delta_{k}(t) \hat{R}_{k}\right|_{\gamma(t)}$, and $\sum_{k=1}^{d}\left|\delta_{k}(t)\right|^{2} \leq \xi^{2}|u-v|^{2}$ for a.e. $t \in[0,1]$. Therefore, $\tilde{d}_{G}(\Phi(u), \Phi(v)) \leq \xi|u-v|$, and the upper bound in (46) is proved.

Next, let $\delta>0$ be such that there is an absolutely continuous path $\gamma:[0,1] \rightarrow$ $g^{-1} \widetilde{B}_{20} g$ such that $\gamma(0)=\Phi(u), \gamma(1)=\Phi(v), \dot{\gamma}(t)=\left.\sum_{k=1}^{d} \gamma_{k}(t) \hat{R}_{k}\right|_{\gamma(t)}$ and $\sum_{k=1}^{d}\left|\gamma_{k}(t)\right|^{2}<\delta^{2}$ for a.e. $t \in[0,1]$. Then, it follows from Lemma $3.4(1)$, that one can write

$$
\dot{\gamma}(t)=\left.\sum_{k=1}^{d} \delta_{k}(t) d \Phi\left(\frac{\partial}{\partial u_{k}}\right)\right|_{\gamma(t)},
$$

and $\sum_{k=1}^{d}\left|\delta_{k}(t)\right|^{2}<\delta^{2} \xi^{2}$ for a.e. $t \in[0,1]$. Hence,

$$
\delta>\xi^{-1} \int_{0}^{1}\left(\sum_{k=1}^{d}\left|\delta_{k}(t)\right|^{2}\right)^{1 / 2} d t \geq \xi^{-1}|u-v|
$$


Therefore, $\xi^{-1}|u-v| \leq \tilde{d}_{G}(\Phi(u), \Phi(v))$, and the lower bound in (46) follows. Statement (I) is therefore proved.

Next, we prove Statement (II). Let $\rho \in\langle 0,1]$ and $u, v, w \in \mathbb{R}^{d}$ be such that $|u|,|v|,|w|<\rho$. Let $\gamma:[0,1] \rightarrow g^{-1} \widetilde{B}_{20} g$ be an absolutely continuous path such that $\gamma(0)=\Phi(u), \gamma(1)=\Phi(v), \dot{\gamma}(t)=\left.\sum_{k=1}^{d} \gamma_{k}(t) \hat{R}_{k}\right|_{\gamma(t)}$, and $\sum_{k=1}^{d}\left|\gamma_{k}(t)\right|^{2} \leq$ $2 \tilde{d}_{G}(\Phi(u), \Phi(v))^{2}$ for a.e. $t \in[0,1]$. Then $\sum_{k=1}^{d}\left|\gamma_{k}(t)\right|^{2} \leq 2 \xi^{2}|u-v|^{2} \leq 8 \xi^{2} \rho^{2}$ for a.e. $t \in[0,1]$. Hence,

$$
\tilde{d}_{G}(\Phi(u), \gamma(t))=\tilde{d}_{G}\left(\Phi(u), \Phi\left(\Phi^{-1}(\gamma(t))\right)\right) \leq 3 \xi \rho,
$$

and $\left|u-\Phi^{-1}(\gamma(t))\right| \leq 3 \xi^{2} \rho$ for a.e. $t \in[0,1]$, by (46). Since $\sum_{k=1}^{d} u_{k} \tilde{a}_{k} \in P_{\rho}^{g}$, it follows that $\left|\Phi^{-1}(\gamma(t))\right| \leq 4 \xi^{2} \rho$ and $\gamma(t) \in g^{-1} \widetilde{B}_{5 \xi^{2} \rho} g$ for all $t \in[0,1]$ if $\rho \leq 4 \xi^{-2}$. Therefore,

$$
\Phi(w) \gamma(t) \in g^{-1} \widetilde{B}_{\rho} g g^{-1} \widetilde{B}_{5 \xi^{2} \rho} g \subseteq g^{-1} \widetilde{B}_{6 K^{2} \xi^{2} \rho} g \subseteq g^{-1} \widetilde{B}_{20} g
$$

for all $t \in[0,1]$, if $\rho \leq \xi^{-2} K^{-2}$. Let $\delta:[0,1] \rightarrow g^{-1} \widetilde{B}_{20} g$ be the absolutely continuous path defined by $\delta(t)=\Phi(w) \gamma(t)$ for all $t \in[0,1]$. Then $\delta(0)=$ $\Phi(w) \Phi(u), \delta(1)=\Phi(w) \Phi(v)$, and $\dot{\delta}(t)=\left.\sum_{k=1}^{d} \gamma_{k}(t) \hat{R}_{k}\right|_{\delta(t)}$ for a.e. $t \in[0,1]$. It follows that one can make $\rho \in\langle 0,1]$ smaller, if necessary, but independent of $g$, such that $\rho \in\left\langle 0, K^{-1}\right]$ and

$$
\tilde{d}_{G}(\Phi(w) \Phi(u), \Phi(w) \Phi(v)) \leq \underset{t \in[0,1]}{\operatorname{ess} \sup }\left(\sum_{k=1}^{d}\left|\gamma_{k}(t)\right|^{2}\right)^{1 / 2}
$$

and, therefore, $\tilde{d}_{G}(\Phi(w) \Phi(u), \Phi(w) \Phi(v)) \leq \tilde{d}_{G}(\Phi(u), \Phi(v))$. Similarly, making $\rho$ smaller again, if necessary, but still independent of $g$, one can show that

$$
\tilde{d}_{G}(\Phi(w) \Phi(u), \Phi(w) \Phi(v)) \geq \tilde{d}_{G}(\Phi(u), \Phi(v)),
$$

and the proof of the corollary is complete.

Step 8. Let $\Omega \subseteq\left(M \cap g^{-1} \widetilde{B}_{20} g\right) \times\left(M \cap g^{-1} \widetilde{B}_{20} g\right)$ be the set of all $(h, k) \in M \times M$ such that $\inf \left\{\delta>0: \Gamma_{\delta}(h, k) \neq \emptyset\right\}<\infty$, where $\Gamma_{\delta}(h, k)$ denotes the set of all absolutely continuous paths $\gamma:[0,1] \rightarrow M \cap g^{-1} \widetilde{B}_{20} g$ such that $\gamma(0)=h, \gamma(1)=k$, $\dot{\gamma}(t)=\left.\sum_{i=1}^{d_{M}} \gamma_{i}(t) \hat{R}_{i}\right|_{\gamma(t)}$, and $\sum_{i=1}^{d_{M}}\left|\gamma_{i}(t)\right|^{2}<\delta^{2}$ for a.e. $t \in[0,1]$. Then the 'metric' $\tilde{d}_{M}: \Omega \rightarrow[0, \infty\rangle$ is defined by $\tilde{d}_{M}(h, k)=\inf \left\{\delta>0: \Gamma_{\delta}(h, k) \neq \emptyset\right\}$ for all $(h, k) \in \Omega$.

For $m_{0} \in M$, define $\Phi_{m_{0}}: \mathbb{R}^{d_{M}} \rightarrow M$ by $\Phi_{m_{0}}(s)=m_{0} \exp \left(\sum_{i=1}^{d_{M}} s_{i} \tilde{a}_{i}\right), s \in \mathbb{R}^{d_{M}}$ Note that $\Phi_{m_{0}}$ is a diffeomorphism from $N$ onto $\Phi_{m_{0}}(N)$, where

$$
N=\left\{s \in \mathbb{R}^{d_{M}}:|s|<200\right\} .
$$


In this step we show that $\tilde{d}_{G}$ and $\tilde{d}_{M}$ are equivalent on $g^{-1} \widetilde{B}_{\varepsilon} g \cap \mathscr{C}$ for all $\varepsilon \in\langle 0, \rho]$ and connected components $\mathscr{C}$ of $M \cap g^{-1} \widetilde{B}_{20} g$, uniformly with respect to $g$, where $\rho \in\left\langle 0, K^{-1}\right]$ is the constant as in Corollary 3.5.

LEMMA 3.6. Let $\mathscr{C}$ be a connected component of $M \cap g^{-1} \widetilde{B}_{20} g$. Then the following two statements hold:

(I) If $\varepsilon \in\langle 0, \rho]$ and $m_{0} \in g^{-1} \widetilde{B}_{\varepsilon} g \cap \mathscr{C}$, then

$$
\xi^{-1}|r-s| \leq \tilde{d}_{M}\left(\Phi_{m_{0}}(r), \Phi_{m_{0}}(s)\right) \leq \xi|r-s|
$$

for all $r, s \in \mathbb{R}^{d_{M}}$ such that $|r|,|s|<3 \varepsilon$ and $\Phi_{m_{0}}(r), \Phi_{m_{0}}(s) \in g^{-1} \widetilde{B}_{\varepsilon} g \cap \mathscr{C}$.

(II) If $\varepsilon \in\langle 0, \rho]$ and $m_{0} \in g^{-1} \widetilde{B}_{\varepsilon} g \cap \mathscr{C}$, then

$$
\tilde{d}_{G}(h, k) \leq \tilde{d}_{M}(h, k) \leq \xi^{2} \tilde{d}_{G}(h, k)
$$

for all $h, k \in g^{-1} \widetilde{B}_{\varepsilon} g \cap \mathscr{C}$.

PROOF. It follows from Lemma 3.3 that there exists an open connected and convex neighbourhood $O \subseteq \mathfrak{m} \cap P_{3 \varepsilon}^{g}$ of 0 such that $\mathscr{C} \cap g^{-1} \widetilde{B}_{\varepsilon} g \subseteq m_{0} \exp (O) \subseteq \mathscr{C}$.

We first prove the upper bound in (48). Let $r, s \in \mathbb{R}^{d_{M}}$ be such that $|r|,|s|<3 \varepsilon$ and $\Phi_{m_{0}}(r), \Phi_{m_{0}}(s) \in g^{-1} \widetilde{B}_{\varepsilon} g \cap \mathscr{C}$. Let $\gamma:[0,1] \rightarrow \mathscr{C}$ be the absolutely continuous path defined by $\gamma(t)=\Phi_{m_{0}}(t s+(1-t) r)$ for all $t \in[0,1]$. Then the path $\gamma$ lies entirely inside $\mathscr{C}$, because $\Phi_{m_{0}}(r), \Phi_{m_{0}}(s) \in m_{0} \exp (O)$, and, by convexity, $\Phi_{m_{0}}(t s+(1-t) r) \in m_{0} \exp (O) \subseteq \mathscr{C}$ for all $t \in[0,1]$. Then it follows from a similar argument as used in the proof of Lemma 3.4, but using (35) instead of (34), that $\tilde{d}_{M}\left(\Phi_{m_{0}}(r), \Phi_{m_{0}}(s)\right) \leq \xi|r-s|$ and the upper bound in (48) follows immediately.

Now, we prove the lower bound in (48). Let $r, s \in \mathbb{R}^{d_{M}}$ be such that $|r|,|s|<3 \varepsilon$ and $\Phi_{m_{0}}(r), \Phi_{m_{0}}(s) \in g^{-1} \widetilde{B}_{\varepsilon} g \cap \mathscr{C}$. Then it is obvious that

$$
\tilde{d}_{M}\left(\Phi_{m_{0}}(r), \Phi_{m_{0}}(s)\right) \geq \tilde{d}_{G}\left(\Phi_{m_{0}}(r), \Phi_{m_{0}}(s)\right) .
$$

Since $m_{0}^{-1}, \Phi_{m_{0}}(r), \Phi_{m_{0}}(s) \in g^{-1} \widetilde{B}_{\varepsilon} g$, there exist $u, v, w \in \mathbb{R}^{d}$ such that $|u|,|v|$, $|w|<\rho, m_{0}^{-1}=\Phi(w), \Phi_{m_{0}}(r)=\Phi(u)$ and $\Phi_{m_{0}}(s)=\Phi(v)$. It follows from Corollary 3.5 (II) that

$$
\begin{aligned}
\tilde{d}_{G}\left(\Phi_{m_{0}}(r), \Phi_{m_{0}}(s)\right) & =\tilde{d}_{G}(\Phi(u), \Phi(v))=\tilde{d}_{G}(\Phi(w) \Phi(u), \Phi(w) \Phi(v)) \\
& =\tilde{d}_{G}\left(m_{0}^{-1} \Phi_{m_{0}}(r), m_{0}^{-1} \Phi_{m_{0}}(s)\right) .
\end{aligned}
$$

Since $P_{3 \varepsilon}^{g} \subseteq P_{20}^{g}$, it follows from the lower bound in (46) that

$$
\tilde{d}_{G}\left(m_{0}^{-1} \Phi_{m_{0}}(r), m_{0}^{-1} \Phi_{m_{0}}(s)\right) \geq \xi^{-1}|r-s|,
$$


and the lower bound in (48) follows. So, Statement (I) is proved.

Moreover, together with the upper bound of (48), the lower bound in (50) implies that

$$
\begin{aligned}
\tilde{d}_{G}\left(\Phi_{m_{0}}(r), \Phi_{m_{0}}(s)\right) & =\tilde{d}_{G}\left(m_{0}^{-1} \Phi_{m_{0}}(r), m_{0}^{-1} \Phi_{m_{0}}(s)\right) \\
& \geq \xi^{-1}|r-s| \geq \xi^{-2} \tilde{d}_{M}\left(\Phi_{m_{0}}(r), \Phi_{m_{0}}(s)\right) .
\end{aligned}
$$

Then the upper bound of (49) is a consequence of Lemma 3.3, and Statement (II) follows immediately, because the lower bound of (49) is trivial.

Step 9. In this step we derive a homothetic contraction result which is inspired by [7, Section 2].

LEMMA 3.7. Let $\mathscr{C}$ be a connected component of $M \cap g^{-1} \widetilde{B}_{20} g$. If $\varepsilon \in\langle 0, \rho]$ and $m_{0} \in \mathscr{C} \cap g^{-1} \widetilde{B}_{\varepsilon} g$, then $|r-s| \geq \xi^{-2}(\rho-\varepsilon)$ for all $r, s \in \mathbb{R}^{d_{M}}$ such that $|r|,|s|<200$, $\Phi_{m_{0}}(r) \in \delta_{M}\left(\mathscr{C} \cap g^{-1} \widetilde{B}_{\varepsilon} g\right)$ and $\Phi_{m_{0}}(s) \in \delta_{M}\left(\mathscr{C} \cap g^{-1} \widetilde{B}_{\rho} g\right)$, where $\delta_{M}$ denotes the boundary in $M$.

PROOF. First, we prove that $\delta_{M}\left(\mathscr{C} \cap g^{-1} \widetilde{\boldsymbol{B}}_{\varepsilon} g\right) \subseteq \delta_{G}\left(g^{-1} \widetilde{\boldsymbol{B}}_{\varepsilon} g\right)$, where $\delta_{G}$ denotes the boundary in $G$. Indeed, let $m \in \delta_{M}\left(\mathscr{C} \cap g^{-1} \widetilde{B}_{\varepsilon} g\right)$, and suppose that $m \notin \delta_{G}\left(g^{-1} \widetilde{B}_{\varepsilon} g\right)$. Then $m \notin \delta_{M}\left(M \cap g^{-1} \widetilde{B}_{\varepsilon} g\right)$, because, if $m \in \delta_{M}\left(M \cap g^{-1} \widetilde{B}_{\varepsilon} g\right)$ and $V$ is a $G$-open neighbourhood of $m$ then $M \cap V$ is an $M$-open neighbourhood of $m$. Hence, there exist $k \in M \cap g^{-1} \widetilde{B}_{\varepsilon} g$ and $l \in M \backslash\left(M \cap g^{-1} \widetilde{B}_{\varepsilon} g\right)$ such that $k, l \in M \cap V$. So, $k \in g^{-1} \widetilde{B}_{\varepsilon} g \cap V, l \in V$, and $l \notin g^{-1} \widetilde{B}_{\varepsilon} g$. Hence, $m \in \delta_{G}\left(g^{-1} \widetilde{B}_{\varepsilon} g\right)$, and this is a contradiction. Therefore, $m$ is an interior point of the connected component $\tilde{\mathscr{C}}$ of $M \cap g^{-1} \widetilde{B}_{\varepsilon} g$, which contains $m$. Since $m \in \delta_{M}\left(\mathscr{C} \cap g^{-1} \widetilde{B}_{\varepsilon} g\right)$, it follows that

$$
\tilde{\mathscr{C}} \cap\left(\mathscr{C} \cap g^{-1} \widetilde{B}_{\varepsilon} g\right) \neq \emptyset .
$$

Therefore, $\tilde{\mathscr{C}} \subseteq \mathscr{C} \cap g^{-1} \widetilde{B}_{\varepsilon} g$, and hence $m$ is an interior point of $\mathscr{C} \cap g^{-1} \widetilde{B}_{\varepsilon} g$, which is a contradiction. Therefore,

$$
\delta_{M}\left(\mathscr{C} \cap g^{-1} \widetilde{B}_{\varepsilon} g\right) \subseteq \delta_{G}\left(g^{-1} \widetilde{B}_{\varepsilon} g\right)=\exp \left(\delta P_{\varepsilon}^{g}\right)
$$

Similarly,

$$
\delta_{M}\left(\mathscr{C} \cap g^{-1} \widetilde{B}_{\rho} g\right) \subseteq \delta_{G}\left(g^{-1} \widetilde{B}_{\rho} g\right)=\exp \left(\delta P_{\rho}^{g}\right) .
$$

Next, let $r, s \in \mathbb{R}^{d_{M}}$ be such that $|r|,|s|<200, \Phi_{m_{0}}(r) \in \delta_{M}\left(\mathscr{C} \cap g^{-1} \widetilde{\boldsymbol{B}}_{\varepsilon} g\right)$ and $\Phi_{m_{0}}(s) \in \delta_{M}\left(\mathscr{C} \cap g^{-1} \widetilde{B}_{\rho} g\right)$. Then there are $u, v \in \mathbb{R}^{d}$ such that $|u|=\varepsilon,|v|=\rho$, $\Phi(u)=\Phi_{m_{0}}(r)$ and $\Phi(v)=\Phi_{m_{0}}(s)$. Then a combination of (46), (48) and (49) gives the estimates

$$
|r-s| \geq \xi^{-1} \tilde{d}_{G}\left(\Phi_{m_{0}}(r), \Phi_{m_{0}}(s)\right)=\xi^{-1} \tilde{d}_{G}(\Phi(u), \Phi(v)) \geq \xi^{-2}|u-v| \geq \xi^{-2}(\rho-\varepsilon),
$$

and the lemma follows. 
Now, fix $\varepsilon \in\left\langle 0,\left(6 \xi^{2}\right)^{-1} \rho\right]$ and a connected component $\mathscr{C}$ of $M \cap g^{-1} \widetilde{B}_{20} g$. Let $m_{0} \in \mathscr{C} \cap g^{-1} \widetilde{B}_{\varepsilon} g$. By Lemma 3.3 there is a convex open neighbourhood $O \subseteq \mathfrak{m} \cap P_{3 \rho}^{g}$ of 0 such that

$$
\mathscr{C} \cap g^{-1} \widetilde{B}_{\rho} g \subseteq m_{0} \exp (O) \subseteq \overline{m_{0} \exp (O)} \subseteq \mathscr{C}
$$

Let $\Gamma_{q}^{m_{0}}: \mathscr{C} \rightarrow M \cap g^{-1} \widetilde{B}_{20} g$ be the homothety with center $m_{0}$ and coefficient $q \in\{0,1]$ (see also [7, Section 2]) defined by $\Gamma_{q}^{m_{0}}\left(\Phi_{m_{0}}(r)\right)=\Phi_{m_{0}}(q r)$ for all $r \in \mathbb{R}^{d_{M}}$ such that $|r|<200$ and $\Phi_{m_{0}}(r) \in \mathscr{C}$.

Let $r \in \mathbb{R}^{d_{M}}$ be such that $|r|<200$ and $\Phi_{m_{0}}(r) \in \delta_{M}\left(m_{0} \exp (O)\right)$. Set

$$
t_{1}=\sup \left\{t \in[0,1]: \Phi_{m_{0}}(t r) \in \mathscr{C} \cap g^{-1} \widetilde{B}_{\varepsilon} g\right\}
$$

Then $\Phi_{m_{0}}\left(t_{1} r\right) \in \delta_{M}\left(\mathscr{C} \cap g^{-1} \widetilde{B}_{\varepsilon} g\right) \subseteq m_{0} \exp \left(\overline{\mathfrak{m} \cap P_{3 \varepsilon}^{g}}\right)$, by Lemma 3.3, and hence $\left|t_{1} r\right| \leq 3 \varepsilon$. Moreover, set

$$
t_{2}=\sup \left\{t \in[0,1]: \Phi_{m_{0}}(t r) \in \mathscr{C} \cap g^{-1} \widetilde{B}_{\rho} g\right\} .
$$

Then $\Phi_{m_{0}}\left(t_{2} r\right) \in \delta_{M}\left(\mathscr{C} \cap g^{-1} \widetilde{B}_{\rho} g\right)$ and $\left|\left(t_{2}-t_{1}\right) r\right| \geq \xi^{-2}(\rho-\varepsilon)$ by Lemma 3.7 . Therefore,

$$
\begin{aligned}
|r| & \geq\left|t_{2} r\right|=\left|\left(t_{2}-t_{1}\right) r\right|+\left|t_{1} r\right| \geq(3 \varepsilon)^{-1}\left|t_{1} r\right| \xi^{-2}(\rho-\varepsilon)+\left|t_{1} r\right| \\
& \geq(3 \varepsilon)^{-1} t_{1}|r| \xi^{-2} \rho+t_{1}|r|\left(1-3^{-1} \xi^{-2}\right) \geq(3 \varepsilon)^{-1} t_{1}|r| \xi^{-2} \rho .
\end{aligned}
$$

So, if one sets $q=6 \xi^{2} \rho^{-1} \varepsilon$, then $1 \geq q>t_{1}$. It follows that

$$
\mathscr{C} \cap g^{-1} \widetilde{B}_{\varepsilon} g \subseteq \Gamma_{q}^{m_{0}}\left(m_{0} \exp (O)\right) \subseteq \Gamma_{q}^{m_{0}}(\mathscr{C}) .
$$

Let $d_{L, m_{0}} m$ denote the image measure on $\Phi_{m_{0}}(N)$, under $\Phi_{m_{0}}$, of the Lebesgue measure $d u_{1} \cdots d u_{d_{M}}$ on $N$, where $N$ is as in (47). Let $\operatorname{Vol}_{M, L, m_{0}}$ be the volume with respect to the measure $d_{L, m_{0}} m$. Set $\widetilde{O}=\left\{u \in \mathbb{R}^{d_{M}}: \sum_{i=1}^{d_{M}} u_{i} \tilde{a}_{i} \in O\right\}$, and let $r: S^{d_{M}-1} \rightarrow$ $\langle 0, \infty\rangle$ be defined by $r(x)=\sup \{r \geq 0: r x \in \widetilde{O}\}$ for $x \in S^{d_{M}-1}$. If $d \sigma(x)$ is the Riemannian measure of $S^{d_{M}-1}$, then

$$
\begin{aligned}
\operatorname{Vol}_{M, L, m_{0}}\left(\Gamma_{q}^{m_{0}}\left(m_{0} \exp (O)\right)\right) & =\int_{S^{d_{M}-1}} \int_{0}^{q r(x)} \tau^{d_{M}-1} d \tau d \sigma(x) \\
& =q^{d_{M}} \int_{S^{d_{M}-1}} \int_{0}^{r(x)} \tau^{d_{M}-1} d \tau d \sigma(x) \\
& =\left(6 \xi^{2} \rho^{-1}\right)^{d_{M}} \varepsilon^{d_{M}} \operatorname{Vol}_{M, L, m_{0}}\left(m_{0} \exp (O)\right) .
\end{aligned}
$$

It follows from (51) and (52) that

$$
\operatorname{Vol}_{M, L, m_{0}}\left(\mathscr{C} \cap g^{-1} \widetilde{B}_{\varepsilon} g\right) \leq C_{\xi, \rho} \varepsilon^{d_{M}} \operatorname{Vol}_{M, L, m_{0}}(\mathscr{C})
$$


for all $\varepsilon \in\left(0, \varepsilon_{0}\right]$, where $C_{\xi, \rho}=\left(6 \xi^{2} \rho^{-1}\right)^{d_{M}}$ and $\varepsilon_{0}=\left(6 \xi^{2}\right)^{-1} \rho \in\langle 0,1]$.

Step 10. In this step we finish the proof of the proposition. First, it is obvious that

$$
\operatorname{Vol}_{M, L, m_{0}}(\mathscr{C})=\operatorname{Vol}_{M, L, e}\left(m_{0}^{-1} \mathscr{C}\right)
$$

and

$$
\operatorname{Vol}_{M, L, m_{i}}\left(\mathscr{C} \cap g^{-1} \widetilde{B}_{\varepsilon} g\right)=\operatorname{Vol}_{M, L, e}\left(m_{0}^{-1}\left(\mathscr{C} \cap g^{-1} \widetilde{B}_{\varepsilon} g\right)\right)
$$

for all $\varepsilon \in\left\langle 0, \varepsilon_{0}\right]$. Hence, it follows from (53) that

$$
\operatorname{Vol}_{M, L, e}\left(m_{0}^{-1}\left(\mathscr{C} \cap g^{-1} \widetilde{B}_{\varepsilon} g\right)\right) \leq C_{\xi, \rho} \varepsilon^{d_{M}} \operatorname{Vol}_{M, L, e}\left(m_{0}^{-1} \mathscr{C}\right)
$$

for all $\varepsilon \in\left\langle 0, \varepsilon_{0}\right]$.

Next, let $v \in \mathbb{R}^{d_{M}}$ be such that $\sum_{i=1}^{d_{M}} v_{i} \tilde{a}_{i} \in P_{200}^{g}$ and $\Phi_{\ell}(v) \in m_{0}^{-1} \mathscr{C}$. Consider the transformation $T_{v}$ from $N$ onto $T_{v}(N)$ given by $T_{v}(u)=\Phi_{e}^{-1}\left(\Phi_{e}(v) \Phi_{e}(u)\right), u \in N$. Then it follows from (36) that the Jacobi determinant $J_{v}$ of the transformation $T_{v}$ in $u=0$ satisfies $v^{-1} \leq J_{v} \leq \nu$. There is a smooth density function $f: m_{0}^{-1} \mathscr{C} \rightarrow\langle 0, \infty\rangle$ such that $d m=f d_{L, e} m$. Since $d m$ is left invariant, it follows that $\nu^{-1} f(e) \leq f(m) \leq$ $v f(e)$ for all $m \in m_{0}^{-1} \mathscr{C}$. Therefore,

$$
\operatorname{Vol}_{M}\left(m_{0}^{-1}\left(\mathscr{C} \cap g^{-1} \widetilde{B}_{\varepsilon} g\right)\right) \leq v^{2} C_{\xi, \rho} \varepsilon^{d_{M}} \operatorname{Vol}_{M}\left(m_{0}^{-1} \mathscr{C}\right)
$$

for all $\varepsilon \in\left\langle 0, \varepsilon_{0}\right]$. Hence, it follows from the left invariance of the Haar measure $d m$ that $\operatorname{Vol}_{M}\left(\mathscr{C} \cap g^{-1} \widetilde{B}_{\varepsilon} g\right) \leq \nu^{2} C_{\xi, \rho} \varepsilon^{d_{M}} \operatorname{Vol}_{M}(\mathscr{C})$ for all $\varepsilon \in\left\langle 0, \varepsilon_{0}\right]$. Considering all connected components $\mathscr{C}$ of $M \cap g^{-1} \widetilde{B}_{20} g$ together, one obtains

$$
\mathrm{Vol}_{M}\left(M \cap g^{-1} \widetilde{B}_{\varepsilon} g\right) \leq \nu^{2} C_{\xi, p} \varepsilon^{d_{M}} \operatorname{Vol}_{M}\left(M \cap g^{-1} \widetilde{B}_{20} g\right)
$$

for all $\varepsilon \in\left\langle 0, \varepsilon_{0}\right]$ and $g \in G$, and (43) holds.

The proof of Proposition 3.1 is complete.

\section{A Nash inequality}

In order to derive kernel bounds for the Laplacian in Section 5 we first need to establish a Nash inequality which we deduce in this section. The results of this section are from [16, Chapter 4].

If $\varphi \in L_{2}(X ; d x) \cap L_{1}(X ; d x)$ and $\psi \in L_{1}(G ; d g)$ then the convolution product $\psi *_{U} \varphi$ is defined by $\psi *_{U} \varphi=\int_{G} \psi(g) U(g) \varphi d g$. Next, by Lemma 2.5 (I)-(II), for all $\varepsilon>0$ one can define the measurable function $\psi_{\varepsilon}^{b}: X \times X \rightarrow[0, \infty)$ by

$$
\psi_{\varepsilon}^{\triangleright}(\dot{g} ; \dot{k})=\int_{M} 1_{B_{r}}\left(g m^{-1} k^{-1}\right) S\left(k, g m^{-1} k^{-1}\right)(\rho(g) \rho(k m))^{-1 / 2} \Delta_{G}\left(m^{-1} k^{-1}\right) d m,
$$

where $g, k \in G$. Moreover, it follows from Lemma 2.5 (III) that $\psi_{\varepsilon}^{b}$ is uniformly bounded on compacta in $X \times X$, so $\int_{X}\left|\psi_{\varepsilon}^{b}(x ; y) \varphi(y)\right| d y<\infty$ for all $x \in X$ and $\varphi \in L_{2}(X ; d x)$ with compact support. 
LEMMA 4.1. If $\varepsilon>0$ and $\varphi \in L_{2}(X ; d x)$ with compact support then

$$
\left(1_{B_{\varepsilon}} *_{U} \varphi\right)(x)=\int_{X} \psi_{\varepsilon}^{b}(x ; y) \varphi(y) d y
$$

for all $x \in X$.

PROOF. First, note that $k \mapsto 1_{B_{\varepsilon}}\left(g k^{-1}\right)(\rho(k) \rho(g))^{-1 / 2} \Delta_{G}\left(k^{-1}\right) \varphi(\dot{k})$ belongs to $L_{1}(G ; \rho d g)$ for all $g \in G, \varepsilon>0$ and $\varphi \in L_{2}(X ; d x)$, since $1_{B_{\varepsilon}}$ has compact support. So, if $g \in G$, then

$$
\begin{aligned}
\int_{X} \psi_{\varepsilon}^{\mathrm{b}}(\dot{g} ; \dot{k}) \varphi(\dot{k}) d \dot{k} \\
\quad=\int_{X}\left(\int_{M} 1_{B_{\varepsilon}}\left(g m^{-1} k^{-1}\right) \frac{S\left(\dot{k}, g m^{-1} k^{-1}\right)}{(\rho(g) \rho(k m))^{1 / 2}} \Delta_{G}\left((k m)^{-1}\right) d m\right) \varphi(\dot{k}) d \dot{k} \\
\quad=\int_{X}\left(\int_{M} 1_{B_{\varepsilon}}\left(g m^{-1} k^{-1}\right) \frac{S\left(\dot{k}, g m^{-1} k^{-1}\right)}{(\rho(g) \rho(k m))^{1 / 2}} \Delta_{G}\left((k m)^{-1}\right) \varphi(k m M) d m\right) d \dot{k} \\
\quad=\int_{G} 1_{B_{\varepsilon}}\left(g k^{-1}\right) S\left(\dot{k}, g k^{-1}\right) \rho(k)^{1 / 2} \rho(g)^{-1 / 2} \Delta_{G}\left(k^{-1}\right) \varphi(\dot{k}) d k \\
\quad=\int_{G} 1_{B_{\varepsilon}}(k) S\left(k^{-1} \dot{g}, k\right) R\left(\dot{g}, k^{-1}\right)^{1 / 2} \varphi\left(k^{-1} \dot{g}\right) d k=\left(1_{B_{\varepsilon}} *_{U} \varphi\right)(\dot{g})
\end{aligned}
$$

by Lemma 2.1 and Lemma 2.2 (III).

Now, we prove a Young type inequality involving $\psi_{\varepsilon}^{b}$. We first need a volume estimate on $\psi_{\varepsilon}^{b}$.

LEMma 4.2. There exists a $C>0$ such that $\psi_{\varepsilon}^{\mathrm{b}}(\dot{g} ; \dot{k}) \leq C \operatorname{Vol}_{G}\left(B_{3 \varepsilon}\right)\left(\operatorname{Vol}_{X}\left(B_{\varepsilon} \dot{k}\right)\right)^{-1}$ for all $g, k \in G$ and $\varepsilon \in\langle 0,1]$.

ProOF. Let $C \geq 1$ be such that $\Delta_{G}(b) \leq C$ for all $b \in B_{1}$. Moreover, let $\varepsilon \in\langle 0,1]$ and $g, k \in G$. We may assume, without loss of generality, that $\psi_{\varepsilon}^{\mathrm{b}}(\dot{g} ; \dot{k}) \neq 0$. Then there exists an $m_{1} \in M$ such that $b=g m_{1}^{-1} k^{-1} \in B_{\varepsilon}$. Let $\tilde{g}=g m_{1}^{-1}$. Then, by Lemma 2.2 (III),

$$
\begin{aligned}
\psi_{\varepsilon}^{\mathrm{b}}(\dot{g} ; \dot{k})= & \psi_{\varepsilon}^{\mathrm{b}}(\dot{\tilde{g}} ; \dot{k}) \\
= & \int_{M} 1_{B_{\varepsilon}}\left(\tilde{g} m^{-1} k^{-1}\right) \frac{S\left(\dot{k}, \tilde{g} m^{-1} k^{-1}\right)}{\left(\rho\left(\tilde{g} m^{-1} k^{-1} k m\right) \rho(k m)\right)^{1 / 2}} \Delta_{G}\left(m^{-1} k^{-1}\right) d m \\
= & \int_{M} 1_{B_{i}}\left(\tilde{g} m^{-1} k^{-1}\right) S\left(\dot{k}, \tilde{g} m^{-1} k^{-1}\right) R\left(\dot{k}, \tilde{g} m^{-1} k^{-1}\right)^{-1 / 2} \\
& \times \rho(k m)^{-1} \Delta_{G}\left(\tilde{g} m^{-1} k^{-1} k^{-1} b^{-1}\right) d m
\end{aligned}
$$




$$
\leq C_{R}^{1 / 2} C_{S} C^{2} \Delta_{G}\left(k^{-1}\right) \int_{M} 1_{B_{\varepsilon}}\left(\tilde{g} m^{-1} k^{-1}\right) \rho(k m)^{-1} d m .
$$

If $m \in M$ and $\tilde{g} m^{-1} k^{-1} \in B_{\varepsilon}$ then $m \in k^{-1} B_{\varepsilon} \tilde{g}=k^{-1} B_{\varepsilon} b k \subseteq k^{-1} B_{2 \varepsilon} k$, because $b \in B_{\varepsilon}$. Therefore,

$$
\psi_{\varepsilon}^{\mathrm{b}}(\dot{g} ; \dot{k}) \leq C_{R}^{1 / 2} C_{S} C^{2} \Delta_{G}\left(k^{-1}\right) \int_{M \cap k^{-1} B_{2} k} \rho(k m)^{-1} d m .
$$

The lemma follows immediately from (56) and (41).

Let $v: X \rightarrow[0, \infty]$ be defined by $v(x)=\inf _{n \in \mathbb{N}_{0}} 2^{n\left(d-d_{M}\right)} \operatorname{Vol}_{X}\left(B_{2^{-n}} x\right), x \in X$. Then, it follows from Proposition 3.1, [13, Annexe 0, Lemme B] and the covering argument as used in Step 1 in Proposition 3.1 that there exists a $C>0$ such that

$$
v(x) \geq C \operatorname{Vol}_{X}(B(x ; 1)),
$$

uniformly for all $x \in X$. Since $x \mapsto 2^{n\left(d-d_{M}\right)} \operatorname{Vol}_{X}\left(B_{2^{-n}} x\right) \quad(x \in X)$ is a continuous function for all $n \in \mathbb{N}_{0}$, it follows that $v$ is a measurable function on $X$.

LEMMA 4.3. There is a $C>0$ such that

$$
\underset{x \in X}{\operatorname{ess} \sup }\left|\int_{X} \psi_{\varepsilon}^{\mathrm{b}}(x ; y) \varphi(y) d y\right| \leq C \varepsilon^{d_{M}}\|\varphi\|_{L_{1}\left(X ; y^{-1} d x\right)}
$$

and

$$
\int_{X}\left|\int_{X} \psi_{\varepsilon}^{b}(x ; y) \varphi(y) d y\right| d x \leq C \varepsilon^{d}\|\varphi\|_{1}
$$

for all $\varepsilon \in\langle 0,1]$ and all $\varphi \in C_{c}(X)$.

Proof. First, if $\varepsilon \in\langle 0,1], y \in X$ and $n \in \mathbb{N}_{0}$ the smallest number such that $2^{-n} \leq \varepsilon$, then $\varepsilon \leq 2^{-n+1}$, and hence $\varepsilon^{d^{-d_{M}}}\left(\operatorname{Vol}_{X}\left(B_{\varepsilon} y\right)\right)^{-1} v(y) \leq\left(2^{n} \varepsilon\right)^{d-d_{M}} \leq 2^{d-d_{M}}$. Then, it follows from Lemma 4.2 and the volume estimates for small balls $B_{\varepsilon}$, that there is a $C>0$ such that

$$
\begin{aligned}
& \underset{x \in X}{\operatorname{ess} \sup }\left|\int_{X} \psi_{\varepsilon}^{\mathrm{b}}(x ; y) \varphi(y) d y\right|=\underset{x \in X}{\operatorname{ess} \sup }\left|\int_{\text {supp } \varphi} \psi_{\varepsilon}^{\mathrm{b}}(x ; y) \varphi(y) v(y) v^{-1}(y) d y\right| \\
& \leq \underset{x \in X}{\operatorname{ess} \sup } \underset{y \in \operatorname{supp} \varphi}{\operatorname{ess} \sup } \psi_{\varepsilon}^{b}(x ; y) v(y)\|\varphi\|_{L_{1}\left(X ; v^{-1} d x\right)} \\
& \leq C \varepsilon^{d} \operatorname{ess} \sup \left(\operatorname{Vol}_{X}\left(B_{\varepsilon} y\right)\right)^{-1} v(y)\|\varphi\|_{L_{1}\left(X ; v^{-1} d x\right)} \\
& \leq C 2^{d-d_{M}} \varepsilon^{d_{M}}\|\varphi\|_{L_{1}\left(X ; v^{-1} d x\right)}
\end{aligned}
$$

for all $\varphi \in C_{c}(X)$ and $\varepsilon \in\langle 0,1]$, and the first statement follows. 
Next, note that $\psi_{\varepsilon}^{D}(\dot{g} ; \dot{k}) \neq 0$ implies that there exists an $m \in M$ such that $g m k^{-1} \in$ $B_{\varepsilon}$, so, $\dot{g} \in B_{\varepsilon} \dot{k}$. Then, by Fubini's theorem, Lemma 4.2 and the volume estimates for small balls $B_{\varepsilon}$, there exist $C_{1}, C_{2}>0$ such that

$$
\begin{aligned}
& \int_{X}\left|\int_{X} \psi_{\varepsilon}^{\mathrm{b}}(x ; y) \varphi(y) d y\right| d x \leq \int_{X}\left(\int_{X} \psi_{\varepsilon}^{\mathrm{b}}(x ; y) d x\right)|\varphi(y)| d y \\
& \leq \int_{X}\left(\int_{B_{\varepsilon} y} \psi_{\varepsilon}^{\mathrm{b}}(x ; y) d x\right)|\varphi(y)| d y \\
& \leq \underset{y \in X}{\operatorname{ess} \sup }\left(\int_{B_{\varepsilon} y} \psi_{\varepsilon}^{b}(x ; y) d x\right)\|\varphi\|_{1} \\
& \leq \underset{y \in X}{\operatorname{ess} \sup } C_{1} \operatorname{Vol}_{X}\left(B_{\varepsilon} y\right) \operatorname{Vol}_{G}\left(B_{3 \varepsilon}\right)\left(\operatorname{Vol}_{X}\left(B_{\varepsilon} y\right)\right)^{-1}\|\varphi\|_{1} \\
& =C_{1} \operatorname{Vol}_{G}\left(B_{3 \varepsilon}\right)\|\varphi\|_{1} \leq C_{2} \varepsilon^{d}\|\varphi\|_{1}
\end{aligned}
$$

for all $\varepsilon \in\langle 0,1]$ and $\varphi \in C_{c}(X)$, and the proof of the lemma is complete.

By interpolation between $L_{1}(X ; d x)$ and $L_{\infty}(X ; d x)$, and between $L_{1}(X ; d x)$ and $L_{1}\left(X ; v^{-1} d x\right)$, we get the following Young type inequality for the function $\psi_{\varepsilon}^{b}$.

LEMMA 4.4. There exists a $C>0$ such that

$$
\left(\int_{X}\left|\int_{X} \psi_{\varepsilon}^{\mathrm{b}}(x ; y) \varphi(y) d y\right|^{2} d x\right)^{1 / 2} \leq C \varepsilon^{\left(d+d_{M}\right) / 2}\|\varphi\|_{L_{1}\left(X ; v^{-1 / 2} d x\right)}
$$

for all $\varepsilon \in\langle 0,1]$ and $\varphi \in C_{c}(X)$.

PROOF. For $\theta \in\langle 0,1\rangle$, let $[\mathscr{X}, \mathscr{Y}]_{\theta}$ denote the complex interpolation space between the Banach spaces $\mathscr{X}$ and $\mathscr{Y}$. It follows, as in [17, Theorem 1.15.3, Step 3], that $L_{1}\left(X ; v^{-1 / 2} d x\right) \subseteq\left[L_{1}(X ; d x), L_{1}\left(X ; v^{-1} d x\right)\right]_{1 / 2}$, and the embedding is continuous. So, there is a $c>0$ such that $\|\varphi\|_{\left(L_{1}(X ; d x),\left.L_{1}\left(X ; v^{-1} d x\right)\right|_{1 / 2} \leq c\|\varphi\|_{L_{1}\left(X ; v^{-1 / 2} d x\right)} \text { for all }\right.}$ $\varphi \in L_{1}\left(X ; v^{-1 / 2} d x\right)$. Then interpolation of the bounds of Lemma 4.3 gives

$$
\begin{aligned}
\left(\int_{X}\left|\int_{X} \psi_{\varepsilon}^{\mathrm{b}}(x ; y) \varphi(y) d y\right|^{2} d x\right)^{1 / 2} & \leq C \varepsilon^{\left(d+d_{M}\right) / 2}\|\varphi\|_{\mid L_{1}(X ; d x), L_{1}\left(X ; v^{-1} d x\right) \|_{1 / 2}} \\
& \leq c C \varepsilon^{\left(d+d_{M}\right) / 2}\|\varphi\|_{L_{1}\left(X ; v^{-1 / 2} d x\right)}
\end{aligned}
$$

for all $\varepsilon \in\langle 0,1]$ and $\varphi \in C_{c}(X)$.

Let $L_{2 ; 1}(X ; d x)=\bigcap_{i=1}^{d} D\left(A_{i}\right) \subseteq L_{2}(X ; d x)$ with norm

$$
\|\varphi\|_{2 ; 1}=\max _{\substack{\alpha \in J(\alpha) \\|\alpha| \leq 1}}\left\|A^{\alpha} \varphi\right\|_{2}
$$

Now we are able to derive the desired Nash inequality. 
PROPOSITION 4.5. There exists a $C>0$ such that

$$
\|\varphi\|_{2} \leq \varepsilon\|\varphi\|_{2 ; 1}+C \varepsilon^{-\left(d-d_{M}\right) / 2}\|\varphi\|_{L_{1}\left(X ; v^{-1 / 2} d x\right)}
$$

for all $\varepsilon>0$ and all $\varphi \in L_{2 ; 1}(X ; d x) \cap L_{1}\left(X ; v^{-1 / 2} d x\right)$.

PROOF. Let $\gamma:[0,1] \rightarrow G$ be an absolutely continuous path from the identity $e$ to $g$ with tangents in the space spanned by $a_{1}, \ldots, a_{d}$. Then there exist $\gamma_{i} \in L_{\infty}([0,1])$ such that $d \psi(\gamma(t)) / d t=\sum_{i=1}^{d} \gamma_{i}(t)\left(\tilde{A}_{i} \psi\right)(\gamma(t))$ for all $\psi \in C_{c}^{\infty}(G)$ and a.e. $t \in[0,1]$. Moreover, recall that

$$
|g|=\inf \int_{0}^{1}\left(\sum_{i=1}^{d} \gamma_{i}(t)^{2}\right)^{1 / 2} d t
$$

where the infimum is taken over all absolutely continuous paths from the identity $e$ to $g \in G$. Therefore, if $L$ denotes the left regular representation of $G$, then

$$
((I-U(g)) \varphi)(x)=\int_{0}^{1} \sum_{i=1}^{d} \gamma_{i}(t)\left(L(\gamma(t)) A_{i} \varphi\right)(x) d t
$$

for all $\varphi \in C_{c}^{\infty}(X)$ and all $x \in X$. Consequently,

$$
\|(I-U(g)) \varphi\|_{2} \leq \int_{0}^{1}\left(\sum_{i=1}^{d} \gamma_{i}(t)^{2}\right)^{1 / 2}\left(\sum_{i=1}^{d}\left\|A_{i} \varphi\right\|_{2}^{2}\right)^{1 / 2} d t
$$

for all $\varphi \in L_{2 ; 1}(X ; d x)$, by the Cauchy-Schwartz inequality. Then, optimalization over all possible paths, gives

$$
\|(I-U(g)) \varphi\|_{2} \leq|g|\left(\sum_{i=1}^{d}\left\|A_{i} \varphi\right\|_{2}^{2}\right)^{1 / 2}
$$

for all $\varphi \in L_{2 ; 1}(X ; d x)$. So, if $\psi \in L_{1}(G ; d g)$ is a positive function with $\|\psi\|_{1}=1$ then

$$
\left\|\varphi-\psi *_{U} \varphi\right\|_{2} \leq \int_{G} \psi(g)|g|\left(\sum_{i=1}^{d}\left\|A_{i} \varphi\right\|_{2}^{2}\right)^{1 / 2} d g
$$

for all $\varphi \in L_{2 ; 1}(X ; d x)$. Since

$$
\|\varphi\|_{2} \leq\left\|\varphi-\left(1_{B_{t}} /\left\|1_{B_{*}}\right\|_{1}\right) *_{U} \varphi\right\|_{2}+\left\|\left(1_{B_{i}} /\left\|1_{B_{*}}\right\|_{1}\right) *_{U} \varphi\right\|_{2}
$$

for all $\varphi \in L_{2}(X ; d x) \cap L_{1}(X ; d x)$, the proposition follows directly from the Lemmas 4.1 and 4.4 , and the fact that there is a $c \geq 1$ such that

$$
c^{-1} \varepsilon^{d} \leq \operatorname{Vol}_{G}\left(B_{\varepsilon}\right)=\left\|1_{B_{i}}\right\|_{1} \leq c \varepsilon^{d}
$$

for all $\varepsilon \in\langle 0,1]$. 


\section{Kernel bounds}

We apply the Nash inequality of Section 4 and the local scaling property of the volume of Section 3 to derive heat kernel upper bounds for higher order strongly elliptic operators. The results are from [16, Chapter 4].

Before we prove Theorem 2.8 we need some preparation. If $\mathscr{X}$ and $\mathscr{Y}$ are Banach spaces then we denote the operator norm of a bounded linear operator $T: \mathscr{X} \rightarrow \mathscr{Y}$ by $\|T\|_{\mathscr{X} \rightarrow \mathscr{Y}}$. In particular, if $\mathscr{X}=L_{r}(X ; d x)$ and $\mathscr{Y}=L_{p}(X ; d x)$, then we abbreviate $\|T\|_{\mathscr{X} \rightarrow \mathscr{Y}}$ by $\|T\|_{r \rightarrow p}$ for a bounded linear operator $T: \mathscr{X} \rightarrow \mathscr{Y}$.

LEMMA 5.1. Let $K$ be a compact subset of $G$. Then there exists a $C_{K}>0$ such that $g^{-1} B_{\varepsilon} g \subseteq B_{C_{K} \varepsilon}$ for all $g \in K$ and $\varepsilon \in\langle 0,1]$.

Proof. By [5, Proposition 6.1], there exist $\varepsilon_{0}>0$ and $C>0$ such that for all $\delta \in\left\langle 0, \varepsilon_{0}\right]$ and $k \in B_{\delta}$ there exist $t_{1}, \ldots, t_{d} \in \mathbb{R}$ such that $k=\exp \left(\sum_{i=1}^{d} t_{i} a_{i}\right)$ and $\left|t_{i}\right| .<C \delta$ for all $i \in\{1, \ldots, d\}$. Suppose $\varepsilon \in\left\langle 0, \varepsilon_{0}\right]$. Since $K$ is compact there exists a $C^{\prime}>0$ such that for all $g \in K$ and $k=\exp \left(\sum_{i=1}^{d} t_{i} a_{i}\right) \in B_{\varepsilon}$, one has

$$
g^{-1} k g=\exp \left(\sum_{i=1}^{d} t_{i} \operatorname{Ad}\left(g^{-1}\right) a_{i}\right)=\exp \left(\sum_{i=1}^{d} s_{i} a_{i}\right),
$$

where $s_{i} \in \mathbb{R}$ with $\left|s_{i}\right|<C^{\prime} \varepsilon$ for all $i \in\{1, \ldots, d\}$, and the lemma follows for small $\varepsilon>0$. Finally, there exists a $\theta>0$ such that $g^{-1} B_{1} g \subseteq B_{\theta}$ for all $g \in K$. Then $g^{-1} B_{\varepsilon} g \subseteq B_{\theta \varepsilon_{0}^{-1} \varepsilon}$ for all $\varepsilon \in\left\langle\varepsilon_{0}, 1\right]$ and $g \in K$.

PROPOSITION 5.2. The following two statements are valid:

(I) If $S^{\Delta}$ denotes the semigroup generated by the Laplacian $\Delta$, then the operator $S_{t}^{\Delta}$ maps $L_{1}\left(X ; v^{-1 / 2} d x\right)$ continuously into $L_{1}\left(X ; v^{-1 / 2} d x\right)$ for all $t>0$. Furthermore, there exist $a, \omega>0$ such that $\left\|S_{t}^{\Delta} \varphi\right\|_{L_{1}\left(X: v^{-1 / 2} d x\right)} \leq a e^{\omega t}\|\varphi\|_{L_{1}\left(X: v^{-1 / 2} d x\right)}$ for all $t>0$ and $\varphi \in L_{1}\left(X ; v^{-1 / 2} d x\right)$.

(II) There exist $a, \omega>0$ such that

$$
\left|\left(\tau, S_{t}^{\Delta} \varphi\right)\right| \leq a t^{-\left(d-d_{M}\right) / 2} e^{\omega t}\|\varphi\|_{L_{1}\left(X ; v^{-1 / 2} d x\right)}\|\tau\|_{L_{1}\left(X ; v^{-1 / 2} d x\right)}
$$

for all $t>0$ and $\varphi, \tau \in L_{1}\left(X ; v^{-1 / 2} d x\right)$.

Proof. First we show that there exist $C, \eta>0$ such that

$$
C^{-1} e^{-\eta|g|} \leq v(g x) v(x)^{-1} \leq C e^{\eta|g|}
$$

for all $x \in X$ and $g \in G$. Note that by Lemma 5.1 there is an $n_{0} \in \mathbb{N}$ such that

$$
\operatorname{Vol}_{X}\left(B_{\varepsilon} g x\right)=\operatorname{Vol}_{X}\left(g g^{-1} B_{\varepsilon} g x\right) \leq \operatorname{Vol}_{X}\left(g B_{C \varepsilon} x\right)
$$


for all $\varepsilon \in\langle 0,1], g \in B_{1}$ and $x \in X$, where $C=2^{n_{0}}$. Then

$$
\operatorname{Vol}_{X}\left(g B_{C \varepsilon} x\right)=\int_{X} 1_{B_{C} x}\left(g^{-1} z\right) d z=\int_{X} 1_{B_{C_{\varepsilon}} x}(z) R(z, g) d z \leq C_{R} \operatorname{Vol}_{X}\left(B_{C \varepsilon} x\right)
$$

for all $\varepsilon \in\langle 0,1], g \in B_{1}$ and $x \in X$. So,

$$
\operatorname{Vol}_{X}\left(B_{\varepsilon} g x\right) \varepsilon^{-\left(d-d_{M}\right)} \leq C_{R} C^{d-d_{M}} \operatorname{Vol}_{X}\left(B_{C \varepsilon} x\right)(C \varepsilon)^{-\left(d-d_{M}\right)}
$$

for all $\varepsilon \in\langle 0,1], g \in B_{1}$ and $x \in X$. Then

$$
\begin{aligned}
v(g x) & \leq \inf _{n \geq n_{0}} 2^{n\left(d-d_{M}\right)} \operatorname{Vol}_{X}\left(B_{2^{-n}} g x\right) \\
& \leq \inf _{n \geq n_{0}} C_{R} 2^{n_{0}\left(d-d_{M}\right)} \operatorname{Vol}_{X}\left(B_{\left.2^{n_{0}-n} X\right) 2^{\left(n-n_{0}\right)\left(d-d_{M}\right)}} \leq C_{R} 2^{n_{0}\left(d-d_{M}\right)} v(x)\right.
\end{aligned}
$$

for all $g \in B_{1}$ and $x \in X$, and the Statement (58) follows from iteration. Hence, $U$ is a continuous representation in the space $L_{1}\left(X ; v^{-1 / 2} d x\right)$. So, the Statement (I) follows directly from general semigroup theory.

Next, let $H_{0}=\Delta+\mu I$, and let $T$ be the semigroup generated by the closure of $H_{0}$, where $\mu$ denotes the ellipticity constant. Since $\overline{H_{0}}$ generates a continuous holomorphic semigroup on $L_{1}\left(X ; v^{-1 / 2} d x\right)$, by [15, Theorem I.5.1], there exist $a, \omega>0$ such that $\left\|T_{t}\right\|_{L_{1}\left(X ; v^{-1 / 2} d x\right) \rightarrow L_{1}\left(X ; v^{-1 / 2} d x\right)} \leq a e^{\omega t}$ for all $t>0$. Let $C$ be the Nash constant as in Proposition 4.5. Let $\varphi \in L_{2}(X ; d x) \cap L_{1}\left(X ; v^{-1 / 2} d x\right)$. Then

$$
\begin{aligned}
\frac{d}{d t}\left\|T_{t} \varphi\right\|_{2}^{2} & =-2 \operatorname{Re}\left(T_{t} \varphi, H_{0} T_{t} \varphi\right) \leq-2 \mu\left\|T_{t} \varphi\right\|_{2 ; 1}^{2} \leq-\frac{2 \mu}{C} \frac{\left\|T_{t} \varphi\right\|_{2}^{2+4 /\left(d-d_{M}\right)}}{\left\|T_{t} \varphi\right\|_{L_{1}\left(X ; v^{-1 / 2} d x\right)}^{4 /\left(d-d_{M}\right)}} \\
& \leq-\frac{2 \mu}{C a^{4 /\left(d-d_{M}\right)} e^{4 \omega t /\left(d-d_{M}\right)}} \frac{\left(\left\|T_{t} \varphi\right\|_{2}^{2}\right)^{1+2 /\left(d-d_{M}\right)}}{\|\varphi\|_{L_{1}\left(X ; v^{-1 / 2} d x\right)}^{\left.4 / d-d_{M}\right)}}
\end{aligned}
$$

for all $t>0$. Therefore,

$$
\begin{aligned}
\frac{d}{d t}\left(\left\|T_{l} \varphi\right\|_{2}^{2}\right)^{-2 /\left(d-d_{M}\right)} & =-\frac{2}{d-d_{M}}\left(\left\|T_{t} \varphi\right\|_{2}^{2}\right)^{-1-2 /\left(d-d_{M}\right)} \frac{d}{d t}\left\|T_{t} \varphi\right\|_{2}^{2} \\
& \geq \frac{4 \mu}{\left(d-d_{M}\right) C a^{4 /\left(d-d_{M}\right)} e^{4\left(\omega t /\left(d-d_{M}\right)\right.}}\|\varphi\|_{L_{1}\left(X ; v^{-1 / 2} d x\right)}^{-4 /\left(d-d_{M}\right)}
\end{aligned}
$$

and by integration,

$$
\begin{aligned}
\left\|T_{t} \varphi\right\|_{2}^{-4 /\left(d-d_{M}\right)} & =\left(\left\|T_{t} \varphi\right\|_{2}^{2}\right)^{-2 /\left(d-d_{M}\right)} \\
& \geq t \frac{4 \mu}{\left(d-d_{M}\right) C a^{4 /\left(d-d_{M}\right)}} e^{-4 \omega t /\left(d-d_{M}\right)}\|\varphi\|_{L_{1}\left(X ; v^{-1 / 2} d x\right)}^{-4 /\left(d-d_{M}\right)}
\end{aligned}
$$

for all $t>0$. Since $L_{2}(X ; d x) \cap L_{1}\left(X ; v^{-1 / 2} d x\right)$ is dense in $L_{1}\left(X ; v^{-1 / 2} d x\right)$, there exist $a, \omega>0$ such that $\left\|S_{t}^{\Delta} \varphi\right\|_{2} \leq a t^{-\left(d-d_{M}\right) / 4} e^{\omega t}\|\varphi\|_{L_{1}\left(X ; v^{-1 / 2} d x\right)}$ for all $t>0$ and $\varphi \in L_{1}\left(X ; v^{-1 / 2} d x\right)$. 
Let $M_{v^{1 / 2}}: L_{1}(X ; d x) \rightarrow L_{1}\left(X ; v^{-1 / 2} d x\right)$ denote the multiplication operator defined by $M_{v^{1 / 2}} \varphi=v^{1 / 2} \varphi$ for all $\varphi \in L_{1}(X ; d x)$. Then there exist $a, \omega>0$ such that $\left\|S_{t}^{\Delta} M_{v^{1 / 2}}\right\|_{1 \rightarrow 2} \leq a t^{-\left(d-d_{M}\right) / 4} e^{\omega t}$ for all $t>0$.

Next, the dual $\left(M_{v^{1 / 2}}\right)^{*}: L_{\infty}\left(X ; v^{-1 / 2} d x\right) \rightarrow L_{\infty}(X ; d x)$ is given by $\left(M_{v^{1 / 2}}\right)^{*} \varphi=\varphi$ for all $\varphi \in L_{\infty}\left(X ; v^{-1 / 2} d x\right)$. Moreover, the dual

$$
\left(S_{1}^{\Delta}\right)^{*}: L_{2}(X ; d x) \rightarrow L_{\infty}\left(X ; v^{-1 / 2} d x\right)
$$

is given by $\left(S_{t}^{\Delta}\right)^{*} \varphi=v^{1 / 2} S_{t}^{\Delta} \varphi$ for all $\varphi \in L_{2}(X ; d x)$ and $t>0$. So, there exist $a^{\prime}, \omega^{\prime}>0$ such that $\left\|v^{1 / 2} S_{t}^{\Delta} \varphi\right\|_{\infty}=\left\|\left(M_{v^{1 / 2}}\right)^{*}\left(S_{t}^{\Delta}\right)^{*} \varphi\right\|_{\infty} \leq a^{\prime} t^{-\left(d-d_{M}\right) / 4} e^{\omega^{\prime} t}\|\varphi\|_{2}$ for all $\varphi \in L_{2}(X ; d x)$ and $t>0$. Therefore, there exist $a, \omega>0$ such that

$$
\left\|v^{1 / 2} S_{t}^{\Delta} v^{1 / 2} \varphi\right\|_{\infty} \leq a^{\prime}\left(2^{-1} t\right)^{-\left(d-d_{M}\right) / 4} e^{2^{-1} \omega^{\prime} t}\left\|S_{t / 2}^{\Delta} v^{1 / 2} \varphi\right\|_{2} \leq a t^{-\left(d-d_{M}\right) / 2} e^{\omega t}\|\varphi\|_{1}
$$

for all $\varphi \in C_{c}(X)$ and $t>0$. Hence,

$$
\begin{aligned}
\left|\left(\tau, S_{t}^{\Delta} \varphi\right)\right| & \leq\|\tau\|_{L_{1}\left(X ; v^{-1 / 2} d x\right)}\left\|v^{1 / 2} S_{t}^{\Delta} v^{1 / 2} v^{-1 / 2} \varphi\right\|_{\infty} \\
& \leq a t^{-\left(d-d_{M}\right) / 2} e^{\omega t}\|\varphi\|_{L_{1}\left(X ; v^{-1 / 2} d x\right)}\|\tau\|_{L_{1}\left(X ; v^{-1 / 2} d x\right)}
\end{aligned}
$$

for all $\varphi, \tau \in L_{1}\left(X ; v^{-1 / 2} d x\right)$ and $t>0$, and Statement (II) follows. The proof of Proposition 5.2 is complete.

Together with the bounds in Proposition 3.1 the bounds of Proposition 5.2 are the main ingredients in the proof of the Gaussian bounds for strongly elliptic operators stated in Theorem 2.8 .

Proof of Theorem 2.8. First, we assume that $S=1$. Our first goal is to show that for all $\alpha, \beta \in J(d)$ and $p \in\{1, \infty\}$ there are $T, a, \omega>0$ such that

$$
\begin{aligned}
& \left|\int_{X} \int_{X}\left(A^{\alpha} R^{\beta} \kappa_{t}\right)(\dot{g} ; \dot{k}) e^{\rho(\psi(\dot{g})-\psi(\dot{k}))} \varphi(\dot{k}) \tau(\dot{g}) d \dot{k} d \dot{g}\right| \\
& \quad \leq a t^{-\left(\dot{d}-d_{M}\right) /(n p)} t^{-(|\alpha|+|\beta|) / n} e^{\omega\left(1+\rho^{n}\right) t}\left\|v^{-1 / 2} \varphi\right\|_{1}\left\|v^{2^{-1}-p^{-1}} \tau\right\|_{p}
\end{aligned}
$$

uniformly for all $t \in\langle 0, T], \varphi, \tau \in C_{c}(X), \rho \in \mathbb{R}$ and real-valued $\psi \in C_{b ; \infty}(X)$ with $\sum_{i=1}^{d}\left|B_{i} \psi\right|^{2} \leq 1$. Since $v$ is positive it suffices to show (59) for all $\tau \geq 0$ only.

The proof is virtually similar to the proof of [6, Proposition 4.2], but differs at essential points. For all $\varphi: X \rightarrow \mathbb{C}$ define the function $\pi^{*} \varphi: G \rightarrow \mathbb{C}$ by $\left(\pi^{*} \varphi\right)(g)=\varphi(\dot{g})$. In [6] the group $M$ was compact and it was sufficient to use the lifting $\pi^{*}$. But now $M$ is not necessarily compact anymore. In order to circumvent convergence problems, we need an alternative lifting. By [10, Lemma 3.2], for all $\tau \in C_{c}(X)$ with $\tau \geq 0$, there exists a continuous function $\pi^{\prime \prime} \tau: G \rightarrow[0, \infty)$ with compact support such that $\tau(\dot{g})=\int_{M}\left(\pi^{\prime \prime} \tau\right)(\mathrm{gm}) d m$ for all $g \in G$. 
Now, let $\alpha, \beta \in J(d)$ and $p \in\{1, \infty\}$. First, the reduction formula of Proposition 2.7 gives

$$
\left(A^{\alpha} R^{\beta} \kappa_{t}\right)(\dot{g} ; \dot{k})=\sum_{(\gamma, \delta) \in L b(\beta)} d_{\delta} \int_{M} \frac{\left(\widetilde{A}^{\alpha} \widetilde{R}^{\gamma} K_{t}\right)\left(g m^{-1} k^{-1}\right)}{(\rho(g) \rho(k m))^{1 / 2}} \Delta_{G}\left(m^{-1} k^{-1}\right) d m
$$

for all $g, k \in G$ and $t>0$. Let $\varphi, \tau \in C_{c}(X), \rho \in \mathbb{R}, \psi \in C_{b ; \infty}(X)$ realvalued, and suppose that $\sum_{i=1}^{d}\left|B_{i} \psi\right|^{2} \leq 1$ and $\tau \geq 0$. By (7) there exist $C>0$ and $\eta>0$ such that $C^{-1} e^{-\eta|g|} \leq \rho(g) \leq C e^{\eta|g|}$ for all $g \in G$. Moreover, there exist $C^{\prime}, \eta^{\prime}>0$ such that $\left(C^{\prime}\right)^{-1} e^{-\eta^{\prime}|g|} \leq \Delta_{G}(g) \leq C^{\prime} e^{\eta^{\prime}|g|}$ for all $g \in G$. Let $\mathscr{K}_{t}(g)=\sum_{(\gamma, \delta) \in L b(\beta)} d_{\delta}\left(\widetilde{A}^{\alpha} \widetilde{R}^{\gamma} K_{t}\right)(g)$. By the Gaussian estimates for $\widetilde{A}^{\alpha} \widetilde{R}^{\gamma} K_{t}$ the function

$$
r \mapsto \mathscr{K}_{t}\left(h r^{-1}\right) e^{\rho\left(\left(\pi^{*} \psi\right)(h)-\left(\pi^{*} \psi\right)(r)\right)}\left(\pi^{*} \varphi\right)(r)\left(\pi^{o} \tau\right)(h) \rho(r)^{-1 / 2} \rho(h)^{1 / 2} \Delta_{G}\left(r^{-1}\right)
$$

belongs to $L_{1}(G ; \rho d g)$ for all $h \in G$ and $t>0$. Therefore, Lemma 2.2 (II) gives

$$
\begin{aligned}
& \int_{G} \mathscr{K}_{l}\left(h r^{-1}\right) e^{\rho\left(\left(\pi^{*} \psi\right)(h)-\left(\pi^{*} \psi\right)(r)\right)}\left(\pi^{*} \varphi\right)(r)\left(\pi^{o} \tau\right)(h)(\rho(r) \rho(h))^{1 / 2} \Delta_{G}\left(r^{-1}\right) d r \\
& \quad=\int_{X} \int_{M} \frac{\mathscr{K}_{t}\left(h m^{-1} k^{-1}\right) \Delta_{G}\left(m^{-1} k^{-1}\right)}{(\rho(k m) \rho(h))^{1 / 2}} e^{\rho\left(\left(\pi^{*} \psi\right)(h)-\psi(k)\right)} \varphi(\dot{k}) d m d \dot{k}\left(\pi^{o} \tau\right)(h) \rho(h) \\
& \quad=\int_{X}\left(A^{\alpha} R^{\beta} \kappa_{l}\right)(\dot{h} ; \dot{k}) e^{\rho\left(\left(\pi^{*} \psi\right)(h)-\psi(\dot{k})\right)} \varphi(\dot{k}) d \dot{k}\left(\pi^{o} \tau\right)(h) \rho(h)
\end{aligned}
$$

for all $h \in G$. Next, the function

$$
h \mapsto\left(\pi^{\circ} \tau\right)(h) e^{\rho\left(\pi^{*} \psi\right)(h)} \int_{X}\left(A^{\alpha} R^{\beta} \kappa_{f}\right)(\dot{h} ; \dot{k}) \varphi(\dot{k}) e^{-\rho \psi(\dot{k})} d \dot{k}
$$

belongs to $L_{1}(G ; \rho d g)$, because the support is a bounded subset of $G$. Therefore, Lemma 2.2 (II) and $(60)$ yield

$$
\begin{aligned}
\int_{G} \int_{G} & \mathscr{K}_{t}\left(h r^{-1}\right) e^{\rho\left(\left(\pi^{*} \psi\right)(h)-\left(\pi^{*} \psi\right)(r)\right)} \\
\times & \left(\pi^{*} \varphi\right)(r)\left(\pi^{o} \tau\right)(h)(\rho(r) \rho(h))^{1 / 2} \Delta_{G}\left(r^{-1}\right) d r d h \\
= & \int_{G} \int_{X}\left(A^{\alpha} R^{\beta} \kappa_{t}\right)(\dot{h} ; \dot{k}) e^{\rho\left(\left(\pi^{*} \psi\right)(h)-\psi(\dot{k})\right)} \varphi(\dot{k}) d \dot{k}\left(\pi^{o} \tau\right)(h) \rho(h) d h \\
= & \int_{X} \int_{M} \int_{X}\left(A^{\alpha} R^{\beta} \kappa_{t}\right)(\dot{g} ; \dot{k}) e^{\rho(\psi(\dot{g})-\psi(\dot{k}))} \varphi(\dot{k}) d \dot{k}\left(\pi^{o} \tau\right)(g n) d n d \dot{g} \\
= & \int_{X} \int_{X}\left(A^{\alpha} R^{\beta} \kappa_{t}\right)(\dot{g} ; \dot{k}) e^{\rho(\psi(\dot{\xi})-\psi(\dot{k}))} \varphi(\dot{k}) d \dot{k} \int_{M}\left(\pi^{o} \tau\right)(g n) d n d \dot{g} \\
= & \int_{X} \int_{X}\left(A^{\alpha} R^{\beta} \kappa_{t}\right)(\dot{g} ; \dot{k}) e^{\rho(\psi(\dot{\xi})-\psi(\dot{k}))} \varphi(\dot{k}) \tau(\dot{g}) d \dot{k} d \dot{g}
\end{aligned}
$$


for all $t>0$.

Secondly, it is clear that $\left(\widetilde{A}_{i}\left(\pi^{*} \psi\right)\right)(g)=\left(B_{i} \psi\right)(\dot{g})$ for all $g \in G$ and $i \epsilon$ $\{1, \ldots, d\}$. So, $\sum_{i=1}^{d}\left|\tilde{A}_{i}\left(\pi^{*} \psi\right)\right|^{2} \leq 1$. It follows that

$$
\left|\left(\pi^{*} \psi\right)(g)-\left(\pi^{*} \psi\right)(h)\right| \leq\left|g h^{-1}\right|
$$

for all $g, h \in G$. From [15, Theorem III.4.8] and an elementary transformation to rewrite the right derivatives in terms of left derivatives and an exponential function, one deduces that there exist $a, b>0$ and $\omega \geq 0$, depending on $\alpha, \beta$, such that

$$
\left|\mathscr{K}_{t}(g)\right| \leq a t^{-(d+|\alpha|+|\beta|) / n} e^{\omega t} e^{-2 b\left(|g|^{n} t^{-1}\right)^{1 /(n-1)}}
$$

for all $g \in G$ and $t>0$. Therefore, it follows from (61) that

$$
\begin{aligned}
\mid \int_{X} \int_{X} & \left(A^{\alpha} R^{\beta} \kappa_{t}\right)(\dot{g} ; \dot{k}) e^{\rho(\psi(\dot{g})-\psi(\dot{k}))} \varphi(\dot{k}) \tau(\dot{g}) d \dot{k} d \dot{g} \mid \\
\leq & \int_{G} \int_{G} a t^{-(d+|\alpha|+|\beta|) / n} e^{\omega t} e^{-2 b\left(\left|h r^{-1}\right|^{n} t^{-1} j^{1 /(n-1)}\right.} e^{\rho\left(\left(\pi^{*} \psi\right)(h)-\left(\pi^{*} \psi\right)(r)\right)} \\
& \times\left|\left(\pi^{*} \varphi\right)(r)\right|\left(\pi^{o} \tau\right)(h)(\rho(r) \rho(h))^{1 / 2} \Delta_{G}\left(r^{-1}\right) d r d h \\
\leq & a t^{-(d+|\alpha|+|\beta|) / n} e^{\omega t} \int_{G} \int_{G} e^{-2 b\left(\left|h r^{-1}\right|^{n} t^{-1}\right)^{1 /(n-1)}} e^{|\rho|\left|h r^{-1}\right|} \\
& \times\left|\left(\pi^{*} \varphi\right)(r)\right|\left(\pi^{o} \tau\right)(h)(\rho(r) \rho(h))^{1 / 2} \Delta_{G}\left(r^{-1}\right) d r d h
\end{aligned}
$$

for all $t>0$. Using the estimate $-b\left(\left|h r^{-1}\right|^{n} t^{-1}\right)^{1 /(n-1)}+\left|\rho \| h r^{-1}\right| \leq \omega_{b} \rho^{n} t$ for all $t>0$ and $h, r \in G$, where $\omega_{b}=b^{-(n-1)}(n-1)^{n-1} n^{-n}$, one deduces that

$$
\begin{aligned}
& \left|\int_{X} \int_{X}\left(A^{\alpha} R^{\beta} \kappa_{t}\right)(\dot{g} ; \dot{k}) e^{\rho(\psi(\dot{g})-\psi(\dot{k}))} \varphi(\dot{k}) \tau(\dot{g}) d \dot{k} d \dot{g}\right| \\
& \leq a t^{-(d+|\alpha|+|\beta|) / n} e^{\omega t+\omega_{b} \rho^{n} t} \\
& \quad \times \int_{G} \int_{G} e^{-b\left(\left|h r^{-1}\right|^{n} t^{-1}\right)^{1 /(n-1)}}\left|\left(\pi^{*} \varphi\right)(r)\right|\left(\pi^{o} \tau\right)(h)(\rho(r) \rho(h))^{1 / 2} \Delta_{G}\left(r^{-1}\right) d r d h
\end{aligned}
$$

for all $t>0$. Therefore, it remains to estimate

$$
t^{-d / n} \int_{G} \int_{G} e^{-b\left(\left|h r^{-1}\right|^{n} t^{-1}\right)^{1 /(n-1)}} \cdot\left|\left(\pi^{*} \varphi\right)(r)\right|\left(\pi^{o} \tau\right)(h)(\rho(r) \rho(h))^{1 / 2} \Delta_{G}\left(r^{-1}\right) d r d h .
$$

Note that the repeated integral is finite by (7) and the fact that the support of $\pi^{\circ} \tau$ is compact.

Thirdly, for all $j \in \mathbb{N}_{0}$, define the annuli $\Omega_{j}$ by

$$
\Omega_{j}=\left\{(r, h) \in G \times G: j \leq\left|h r^{-1}\right|^{n} t^{-1}<j+1\right\} .
$$


Let $d \nu_{j}(r, h)$ denote the measure on $\Omega_{j}$ induced by $d r d h$. Then

$$
\begin{aligned}
& \int_{G} \int_{G} \frac{\left|\left(\pi^{*} \varphi\right)(r)\right|\left(\pi^{o} \tau\right)(h)}{t^{d / n} e^{b\left(\left|h r^{-1}\right|^{n^{-1}}\right)^{1 /(n-1)}}}(\rho(r) \rho(h))^{1 / 2} \Delta_{G}\left(r^{-1}\right) d r d h \\
& =\sum_{j=0}^{\infty} \int_{\Omega_{j}} \frac{\left|\left(\pi^{*} \varphi\right)(r)\right|\left(\pi^{0} \tau\right)(h)}{t^{d / n} e^{b\left(\left|h r^{-1}\right| t^{-1}\right)^{1 /(n-1)}}}(\rho(r) \rho(h))^{1 / 2} \Delta_{G}\left(r^{-1}\right) d \nu_{j}(r, h) \\
& \leq \sum_{j=0}^{\infty} \frac{s_{j}^{d / 2} e^{((j+1) t)^{2 / n} s_{j}^{-1}}}{t^{d / n} e^{b j !(u-1)}} \int_{\Omega_{j}} \frac{\left|\left(\pi^{*} \varphi\right)(r)\right|\left(\pi^{o} \tau\right)(h)}{s_{j}^{d / 2} e^{\left|h r^{-1}\right|^{2} s_{j}^{-1}}}(\rho(r) \rho(h))^{1 / 2} \Delta_{G}\left(r^{-1}\right) d \nu_{j}(r, h) \\
& \left.\leq \sum_{j=0}^{\infty} \frac{s_{j}^{d / 2} e^{((j+1) t)^{2 / n} s_{j}^{-1}}}{t^{d / n} e^{b j ! /(n-1)}} \int_{G} \int_{G}^{\left|\left(\pi^{*} \varphi\right)(r)\right|\left(\pi^{o} \tau\right)(h)} \frac{s_{j}^{d / 2} e^{\left|h r^{-1}\right|^{2} s_{j}^{-1}}}{(\rho)}(r) \rho(h)\right)^{1 / 2} \Delta_{G}\left(r^{-1}\right) d r d h,
\end{aligned}
$$

for all $t>0$, where $s_{j}>0$ for all $j \in \mathbb{N}_{0}$.

Fourthly, let $K^{\Delta}$ and $\kappa^{\Delta}$ denote the Lie group kernel and reduced heat kernel of the semigroup $S^{\Delta}$ generated by the Laplacian $\Delta=-\sum_{i=1}^{d} A_{i}^{2}$. By [15, Theorem III.5.1], there exist $a, c>0$ and $\omega_{1} \geq 0$ such that $s^{-d / 2} e^{-|g|^{2} s^{-1}} \leq a e^{\omega_{1} s} K_{c s}^{\Delta}(g)$ for all $s>0$ and $g \in G$. Then, it follows from a similar reduction procedure as used in (61) that

$$
\begin{aligned}
& \int_{G} \int_{G} \frac{\left|\left(\pi^{*} \varphi\right)(r)\right|\left(\pi^{o} \tau\right)(h)}{s_{j}^{d / 2} e^{\left.\left|h r^{-1}\right|\right|_{j} ^{-1}}}(\rho(r) \rho(h))^{1 / 2} \Delta_{G}\left(r^{-1}\right) d r d h \\
& \quad \leq a e^{\omega_{1} s_{j}} \int_{G} \int_{G}\left|\left(\pi^{*} \varphi\right)(r)\right|\left(\pi^{o} \tau\right)(h) K_{c s_{j}}^{\Delta}\left(h r^{-1}\right)(\rho(r) \rho(h))^{1 / 2} \Delta_{G}\left(r^{-1}\right) d r d h \\
& \quad=a e^{\omega_{1} s_{j}} \int_{X} \int_{X} \kappa_{r s_{j}}^{\Delta}(x ; y)|\varphi(y)| d y \tau(x) d x=a e^{\omega_{1} s_{j}}\left(\tau, S_{c s_{j}}^{\Delta}|\varphi|\right),
\end{aligned}
$$

where $s_{j}>0$ for all $j \in \mathbb{N}_{0}$. Note that the first equality is valid, since $\tau$ is positive. Then, by the bounds of Proposition 5.2, there exist $a>0$ and $\omega \geq 0$ such that

$$
\begin{aligned}
& \int_{G} \int_{G} \frac{\left|\left(\pi^{*} \varphi\right)(r)\right|\left(\pi^{o} \tau\right)(h)}{t^{d / n} e^{b\left(\left|h r^{-1}\right| f^{-1}\right)^{1 /(n-1)}}(\rho(r) \rho(h))^{1 / 2} \Delta_{G}\left(r^{-1}\right) d r d h} \\
& \quad \leq a \sum_{j=0}^{\infty} \frac{s_{j}^{d / 2} e^{((j+1) t)^{2 / n_{j}-1}} e^{\omega s_{j}}}{t^{d / n} e^{b j /(n-1)} s_{j}^{\left(d-d_{M}\right) /(2 p)}}\left\|v^{-1 / 2} \varphi\right\|_{I}\left\|v^{2^{-1}-p^{-1}} \tau\right\|_{p},
\end{aligned}
$$

uniformly for all $s_{1}, s_{2}, \ldots>0, t>0$ and $\varphi, \tau \in C_{r}(X)$ with $\tau \geq 0$.

If we now take the choice $s_{j}=(j+1)^{1 /(n-1)} t^{2 / n}>0$ for all $j \in \mathbb{N}_{0}$, then

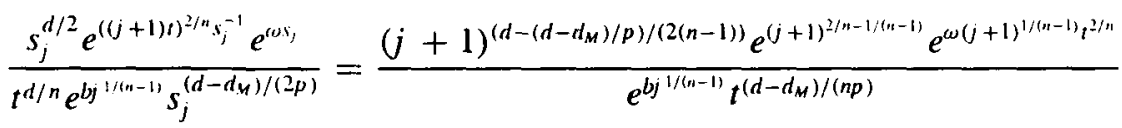

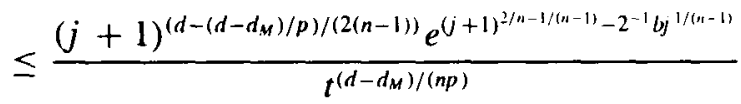


for all $j \geq 1$ and $t>0$ with $2 \omega t^{2 / n} \leq 2^{-1} b$. Moreover, if $j=0$, then

$$
\begin{aligned}
\frac{s_{j}^{d / 2} e^{((j+1) t)^{2 / n} s_{j}^{-1}} e^{\omega s_{j}}}{t^{d / n} e^{b_{j}^{1 /(n-1)}} s_{j}^{\left(d-d_{M}\right) /(2 p)}} & =\frac{(j+1)^{\left(d-\left(d-d_{M}\right) / p\right) /(2(n-1))} e^{(j+1)^{2 / n-1 /(n-1)}} e^{\omega(j+1)^{1 /(n-1) t^{2 / n}}}}{e^{b j j^{1 /(n-1)}} t^{\left(d-d_{M}\right) /(n p)}} \\
& \leq e^{1+4^{-1} b} t^{-\left(d-d_{M}\right) /(n p)}
\end{aligned}
$$

for all $t>0$ with $2 \omega t^{2 / n} \leq 2^{-1} b$.

Since $2 / n-1 /(n-1)<1 /(n-1)$, it follows that

$$
M=e^{1+4^{-1} b}+\sum_{j=1}^{\infty}(j+1)^{\left(d-\left(d-d_{M}\right) / p\right) /(2(n-1))} e^{(j+1)^{2 / n-1 /(n-1)}-2^{-1} b j^{1 /(n-1)}}<\infty,
$$

and

$$
\begin{gathered}
\int_{G} \int_{G} \frac{\left|\left(\pi^{*} \varphi\right)(r)\right|\left(\pi^{o} \tau\right)(h)}{t^{d / n} e^{b\left(\left|h r^{-1}\right| t^{-1}\right)^{1 /(n-1)}}(\rho(r) \rho(h))^{1 / 2} \Delta_{G}\left(r^{-1}\right) d r d h} \\
\leq a M t^{-\left(d-d_{M}\right) /(n p)}\left\|v^{-1 / 2} \varphi\right\|_{1}\left\|v^{2^{-1}-p^{-1}} \tau\right\|_{p}
\end{gathered}
$$

for all $t \in\left\langle 0,\left((4 \omega)^{-1} b\right)^{n / 2}\right]$ and $\varphi, \tau \in C_{c}(X)$ with $\tau \geq 0$. Hence, (59) is valid.

Let $U_{\rho}$ be the multiplication operator with $e^{-\rho \psi}$ and let $\beta_{*}=\left(i_{k}, \ldots, i_{1}\right) \in J(d)$, whenever $\beta=\left(i_{1}, \ldots, i_{k}\right) \in J(d)$. Then, it follows from (59) with $p=1$ that, for all $\alpha, \beta \in J(d)$ there exist $c_{\alpha, \beta}, T_{\alpha, \beta}, \omega_{\alpha, \beta}>0$ such that

$$
\begin{aligned}
& \left\|U_{\rho}^{-1} v^{1 / 2} A^{\alpha} S_{t} A^{\beta *} U_{\rho}\right\|_{L_{1}\left(X ; v^{-1 / 2} d x\right) \rightarrow L_{\infty}(X ; d x)} \\
& \quad=\left\|U_{\rho}^{-1} v^{1 / 2} A^{\alpha} S_{t} A^{\beta *} v^{1 / 2} U_{\rho}\right\|_{1 \rightarrow \infty} \leq c_{\alpha, \beta} t^{-\left(|\alpha|+|\beta|+d-d_{M}\right) / n} e^{\omega_{\alpha, \beta}\left(1+\rho^{n}\right) t},
\end{aligned}
$$

uniformly for all $t \in\left\langle 0, T_{\alpha, \beta}\right], \rho \in \mathbb{R}$, and real-valued $\psi \in C_{b ; \infty}(X)$ such that $\sum_{i=1}^{d}\left|B_{i} \psi\right|^{2} \leq 1$. Moreover, it follows from (59) with $p=\infty$ that, for all $\alpha, \beta \in J(d)$ there exist $c_{\alpha, \beta}^{\prime}, T_{\alpha, \beta}^{\prime}, \omega_{\alpha, \beta}^{\prime}>0$ such that

$$
\begin{aligned}
\left\|U_{\rho}^{-1} A^{\alpha} S_{t} A^{\beta *} U_{\rho}\right\|_{L_{1}\left(X ; v^{-1 / 2} d x\right) \rightarrow L_{1}\left(X ; v^{-1 / 2} d x\right)} \\
\quad=\left\|U_{\rho}^{-1} v^{-1 / 2} A^{\alpha} S_{t} A^{\beta_{*}} v^{1 / 2} U_{\rho}\right\|_{1 \rightarrow 1} \\
\quad \leq c_{\alpha, \beta}^{\prime} t^{-(|\alpha|+|\beta|) / n} e^{\omega_{\alpha, \beta}^{\prime}\left(1+\rho^{n}\right) t}
\end{aligned}
$$

for all $t \in\left\langle 0, T_{\alpha, \beta}^{\prime}\right], \rho \in \mathbb{R}$ and real-valued $\psi \in C_{b ; \infty}(X)$ with $\sum_{i=1}^{d}\left|B_{i} \psi\right|^{2} \leq 1$. In particular, there are $a, T, \omega>0$ such that

$$
\left\|U_{\rho}^{-1} S_{t} U_{\rho}\right\|_{L_{1}\left(X: v^{-1 / 2} d x\right) \rightarrow L_{1}\left(X ; v^{-1 / 2} d x\right)}=\left\|U_{\rho}^{-1} v^{-1 / 2} S_{t} v^{1 / 2} U_{\rho}\right\|_{1 \rightarrow 1} \leq a e^{\omega\left(1+\rho^{n}\right) t}
$$

for all $t \in\langle 0, T], \rho \in \mathbb{R}$ and real-valued $\psi \in C_{b ; \infty}(X)$ with $\sum_{i=1}^{d}\left|B_{i} \psi\right|^{2} \leq 1$. Hence, by iteration, and enlarging $a, \omega$ if necessary, it follows that (64) is valid for all $t>0$, 
$\rho \in \mathbb{R}$ and real-valued $\psi \in C_{b ; \infty}(X)$ with $\sum_{i=1}^{d}\left|B_{i} \psi\right|^{2} \leq 1$. Then the semigroup property gives

$$
\begin{aligned}
&\left\|U_{\rho}^{-1} v^{1 / 2} A^{\alpha} S_{t} A^{\beta \cdot} \cdot v^{1 / 2} U_{\rho}\right\|_{1 \rightarrow \infty} \\
& \leq\left\|U_{\rho}^{-1} v^{1 / 2} A^{\alpha} S_{t_{1}} U_{\rho}\right\|_{L_{1}\left(X ; v^{-1 / 2} d x\right) \rightarrow L_{\infty}(X ; d x)}\left\|U_{\rho}^{-1} S_{t_{2}} U_{\rho}\right\|_{L_{1}\left(X ; v^{-1 / 2} d x\right) \rightarrow L_{1}\left(X ; v^{-1 / 2} d x\right)} \\
& \times\left\|U_{\rho}^{-1} S_{l_{3}} A^{\beta \cdot} U_{\rho}\right\|_{L_{1}\left(X ; v^{-1 / 2} d x\right) \rightarrow L_{1}\left(X ; v^{-1 / 2} d x\right)}
\end{aligned}
$$

for all $t_{1}, t_{2}, t_{3}>0$ with $t_{1}+t_{2}+t_{3}=t, \rho \in \mathbb{R}$ and real-valued $\psi \in C_{b ; \infty}(X)$ with $\sum_{i=1}^{d}\left|B_{i} \psi\right|^{2} \leq 1$. Then, for all $\alpha, \beta \in J(d)$ there exist $a, \omega>0$ such that

$$
\left\|U_{\rho}^{-1} v^{1 / 2} A^{\alpha} S_{t} A^{\beta \cdot} v^{1 / 2} U_{\rho}\right\|_{1 \rightarrow \infty} \leq a t^{-\left(|\alpha|+|\beta|+d-d_{M}\right) / n} e^{\omega\left(1+\rho^{n}\right) t}
$$

for all $t>0, \rho \in \mathbb{R}$ and real-valued $\psi \in C_{b ; \infty}(X)$ with $\sum_{i=1}^{d}\left|B_{i} \psi\right|^{2} \leq 1$. Minimizing first over $\psi$, and then over $\rho$, one observes that for all $\alpha, \beta \in J(d)$ there exists a $b>0$ such that

$$
\left|\left(A^{\alpha} R^{\beta} \kappa_{t}\right)(\dot{g} ; \dot{k})\right| \leq a v(\dot{g})^{-1 / 2} v(\dot{k})^{-1 / 2} t^{-\left(|\alpha|+|\beta|+d-d_{M}\right) / n} e^{\omega t} e^{-b\left(d\left(\dot{g} ; k^{n} t^{-1}\right)^{1 /(n-1)}\right.}
$$

for all $g, k \in G$ and $t>0$.

Finally, it follows from (57) that we may replace $v(\dot{g})$ and $v(\dot{k})$ by $\operatorname{Vol}_{X}(B(\dot{g} ; 1))$ and $\operatorname{Vol}_{X}(B(k ; 1))$, respectively, in the bounds $(65)$, and the proof of Theorem 2.8 is completed for $S=1$. If $S \neq 1$ then the proof is almost the same. Note that we get an extra term $S\left(\dot{k}, \mathrm{gm}^{-1} k^{-1}\right)$ in the reduced expression but this term does not cause any convergence problems in the above argument, because, by (12),

$$
\left|S\left(\dot{k}, g m^{-1} k^{-1}\right)\right| \leq C^{\prime} e^{\eta^{\prime}(|g|+|m|+|k|)}
$$

for all $g, k \in G$ and $m \in M$. Moreover we obtain similar estimates as (62), but now with the Laplacian semigroup $S^{\Delta}$, where $\Delta$ is the Laplacian associated to the representation $U$ defined by the cocycle $|S|$ instead of $S=1$. Fortunately, for this semigroup the estimates in Proposition 5.2 still hold, and the theorem follows immediately.

Acknowledgements The author wishes to thank A. F. M. ter Elst for the many fruitful discussions, comments and suggestions.

\section{References}

[1] W. Arendt and A. V. Bukhvalov, 'Integral representations of resolvents and semigroups', Forum Math. 6 (1994), 111-135. 
[2] D. G. Aronson, 'Bounds for the fundamental solution of a parabolic equation', Bull. Amer. Math. Soc. 73 (1967), 890-896.

[3] E. B. Davies, Heat kernels and spectral theory, Cambridge Tracts in Mathematics 92 (Cambridge University Press, Cambridge, 1989).

[4] A. F. M. ter Elst and D. W. Robinson, 'Subcoercive and subelliptic operators on Lie groups: variable coefficients', Publ. RIMS. Kyoto Univ. 29 (1993), 745-801.

[5] —_, 'Weighted subcoercive operators on Lie groups', J. Funct. Anal. 157 (1998), 88-163.

[6] A. F. M. ter Elst and C. M. P. A. Smulders, 'Reduced heat kernels on homogeneous spaces', J. Operator Theory 42 (1999), 269-304.

[7] A. Grigor'yan, 'The heat equation on noncompact Riemannian manifolds', Math. USSR Sbornik 72 (1992), 47-77.

[8] —_, 'Heat kernel upper bounds on a complete non-compact manifold', Rev. Mat. Iberoamericana 10 (1994), 395-452.

[9] D. S. Jerison and A. Sánchez-Calle, 'Estimates for the heat kernel for a sum of squares of vector fields', Ind. Univ. Math. J. 35 (1986), 835-854.

[10] T. H. Koomwinder, Representations of locally compact groups with applications Part II, MC Syllabus 38.2 (Mathematical Centre, Amsterdam, 1979).

[11] P. Li and S. T. Yau, 'On the upper estimate of the heat kernel of a complete Riemannian manifold', Amer. J. Math. 103 (1981), 1021-1063.

[12] N. Lohoué and S. Mustapha, 'Sur les transformées de riesz sur les groupes de Lie moyennables et sur certains espaces homogènes', Canad. J. Math. 50 (1998), 1090-1 104.

[13] P. Maheux, Analyse et géometrie sur les espaces homogènes (Ph.D. Thesis, Université de Paris VI, Paris, 1991).

[14] _-, 'Estimations du noyau de la chaleur sur les espaces homogènes', J. Geom. Anal. 8 (1998), 65-96.

[15] D. W. Robinson, Elliptic operators and Lie groups, Oxford Mathematical Monographs (Oxford University Press, Oxford, 1991).

[16] C. M. P. A. Smulders, Reduced heat kernels on homogeneous spaces (Ph.D. Thesis, Eindhoven University of Technology, Eindhoven, the Netherlands, 2000).

[17] H. Triebel, Interpolation theory, function spaces, differential operators (North-Holland, Amsterdam, 1978).

[18] N. T. Varopoulos, 'The heat kernel on Lie groups', Rev. Math. Iberoamericana 12 (1996), 147-186.

Department of Mathematics and Comp. Sci.

Eindhoven University of Technology

P.O. Box 513

$5600 \mathrm{MB}$ Eindhoven

The Netherlands

e-mail: camsmul@cs.com 